\title{
Poincaré's inequality and global solutions of a nonlinear parabolic equation
}

by

\section{Philippe SOUPLET and Fred B. WEISSLER}

Laboratoire Analyse Géométrie et Applications, UMR CNRS 7539, Institut Galilée, Université Paris-Nord, 93430 Villetaneuse, France e-mail: souplet@math.univ-paris13.fr ; weissler@math.univ-paris13.fr

\begin{abstract}
We study the equation $u_{t}-\Delta u=u^{p}-\mu|\nabla u|^{q}, t \geq 0$ in a general (possibly unbounded) domain $\Omega \subset \mathbb{R}^{N}$. When $q \geq p$, we show a close connection between the Poincaré inequality and the boundedness of the solutions. To be more precise, if $q>p$ (or $q=p$ and $\mu$ large enough), we prove global existence of all solutions for any domain $\Omega$ where the Poincaré inequality is valid. When $\mu$ is large enough, all solutions are bounded and decay exponentially to zero. Conversely, if $\Omega$ contains arbitrarily large balls (if $N \leq 2$ and $\Omega$ is finitely connected, this means precisely that the Poincare inequality does not hold), then there always exist unbounded solutions. Moreover, if $\Omega=\mathbb{R}^{N}$, there exist global solutions which blow-up at every point in infinite time. Various qualitative properties of the solutions are also obtained.

(C) 1999 L'Association Publications de l'Institut Henri Poincaré. Published by Elsevier B.V. All rights reserved

Key words: nonlinear parabolic equations, gradient term, global existence, bounded solutions, Poincaré inequality, blow-up, critical exponent, exponential decay.
\end{abstract}

RÉSUMÉ. - Nous étudions l'équation $u_{t}-\Delta u=u^{p}-\mu|\nabla u|^{q}, t \geq 0$ dans un domaine $\Omega \subset \mathbb{R}^{N}$ général, éventuellement non borné. Lorsque $q \geq p$, nous montrons l'existence d'un lien étroit entre l'inégalité de Poincaré et le caractère borné des solutions. Plus précisément, si $q>p$ (ou si $q=p$ et $\mu$ est assez grand), nous prouvons l'existence globale de toutes les solutions, dans tout domaine $\Omega$ ou l'inégalité de Poincaré est vérifiée.

AMS Classification: 35 K 60, 35 B 35, 35 B 60 .

Annales de l'Institut Henri Poincaré - Analyse non linéaire - 0294-1449 
Lorsque $\mu$ est suffisamment grand, toutes les solutions sont bornées et tendent exponentiellement vers zéro. Inversement, si $\Omega$ contient des boules de rayon arbitrairement grand (lorsque $N \leq 2$ et $\Omega$ est finiment connexe, ccla signific exactement que l'inćgalité de Poincaré n'a pas licu), alors il existe toujours des solutions non bornées. De plus, si $\Omega=\mathbb{R}^{N}$, il existe des solutions globales qui explosent en tout point en temps infini. Diverses propriétés qualitatives des solutions globales sont également obtenues.

(C) 1999 L'Association Publications de l'Institut Henri Poincaré. Published by Elsevier B.V. All rights reserved

\section{INTRODUCTION AND MAIN RESULTS}

We consider the following parabolic equation:

$$
\begin{aligned}
& u_{t}-\Delta u=u^{p}-\mu|\nabla u|^{q}, \quad t>0, \quad x \in \Omega, \\
& u(t, x)=0, \quad t>0, \quad x \in \partial \Omega, \\
& u(0, x)=\phi(x) \geq 0, \quad x \in \Omega,
\end{aligned}
$$

where $p, q>1, \mu \geq 0$, and $\Omega$ is a (possibly unbounded) regular domain in $\mathbb{R}^{N}$. (By a regular domain, we mean a uniformly regular domain of class $C^{2}$ in the sense of Browder [B] and Amann [Am].) The problem (1.1)(1.3) admits a unique, local in time solution $u \geq 0$, for any $\phi \in W_{0}^{1, s}(\Omega)$, $\phi \geq 0$, with $s$ large enough $(\max (N p, N q)<s<\infty)$. We refer to the Appendix A for a precise definition and local properties of solutions. We denote by $T^{*}=T^{*}(\phi), 0<T^{*} \leq \infty$, the maximal existence time of the solution.

This equation was introduced by Chipot and the second author [CW] in order to investigate the effect of a damping term on global existence or nonexistence. On the other hand, the first author ([S2]) proposed a model in population dynamics, where (1.1)-(1.3) describes the evolution of the population density of a biological species, under the effect of certain natural mechanisms.

Several authors have studied the existence of nonglobal positive solutions for the problem (1.1)-(1.3) and have given various sufficient conditions for blow-up under certain assumptions on $p, q, \mu, N$ and $\Omega$ ([CW], [AW], [KP], [F], [Q1], [Q2], [S1], [S2], [STW]). Unifying and improving these results, the authors of the present paper proved the following ([SW, Corollary 3]): 
THEOREM A. - Let $p>q \geq 1$ and $\mu>0$. Let $\psi \in W_{0}^{1, s}(\Omega), \psi \geq 0$, $\psi \not \equiv 0$. Then there exists some $k_{0}>0$ (depending on $\psi$ ) such that for all $k>k_{0}$, the solution of (1.1)-(1.3) with initial data $\phi=k \psi$ blows-up in finite time in $W^{1, s}$ norm.

This theorem is a consequence of a general result of [SW], valid for a wide class of nonlinear parabolic equations of the form:

$$
u_{t}+L(u) u=F(u, \nabla u)
$$

It relies on a method of blowing-up self-similar subsolutions introduced in [SW]. In the case of equation (1.1), both the result and the proof provided, as an important advantage, a unified treatment for the general case $q<p$, independent of all the technical restrictions that had to be imposed in the previous blow-up studies for this equation.

On the other hand, when $q \geq p$ and $\Omega$ is bounded, global existence for all nonnegative initial data was proved in [F], [Q2]. In particular, this combined with Theorem A established the following conjecture, in the case of bounded domains:

The critical blow-up exponent for problem (1.1)-(1.3) is $q=p$,

in the sense that blow-up can occur if and only if $q<p$ (see [Q1, p. 413]).

The initial motivation of the present paper is to study this conjecture in the case of unbounded domains. We shall prove that finite time blow-up cannot occur for $q>p$ (or $q=p$ and $\mu$ large enough), whenever the Poincaré inequality is valid in $W_{0}^{1, q}(\Omega)$, that is:

$$
\|v\|_{L^{q}(\Omega)} \leq C_{q}(\Omega)\|\nabla v\|_{L^{q}(\Omega)}, \forall v \in W_{0}^{1, q}(\Omega) .
$$

Moreover, for $\mu$ large enough, all solutions are globally bounded and decay exponentially to 0 , provided the Poincaré inequality is also valid in $H_{0}^{1}(\Omega)$.

Although we are still unable to prove or to exclude blow-up for $q \geq p$ in a general unbounded domain, we will see that the assumption on the Poincaré incquality is not artificial and turns out to play a significant role in the problem. To this end, let us first recall that the inradius of $\Omega$ is defined as:

$$
\rho(\Omega)=\sup \{r>0 ; \Omega \text { contains a ball of radius } r\} .
$$

For finitely connected domains in dimension $N=2$, the finiteness of the inradius is known to be equivalent to the validity of the Poincare inequality Vol. $16, n^{\circ} 3-1999$. 
in $H_{0}^{1}(\Omega)$ (Hayman [H], Osserman [O]), or -in other terms- to the fact that $-\Delta$ has a smallest eigenvalue, which is positive, where $\Delta$ is the Laplace operator in $H_{0}^{1}(\Omega)$. (This is also obviously true for any interval in dimension $N=1$.) We shall prove that if $\rho(\Omega)=\infty$, then there exist (possibly global) unbounded solutions for all $q \geq p$ and $\mu>0$.

By combining these results, in dimension 1 or 2 and under some technical assumptions, we obtain a characterization in terms of the Poincaré inequality of the domains in which all solutions are global and bounded (for $q \geq p$ and $\mu$ large).

Let us now state our main results in a more precise form. Recall that the exponent $s$ satisfies $\max (N p, N q)<s<\infty$.

THEOREM 1 (global existence). - Let $\Omega$ be a uniformly regular domain of class $C^{2}$ in $\mathbb{R}^{N}, q \geq p>1$ and $\mu>0$ (with $\mu$ large enough if $q=p)$. Assume that the Poincaré inequality holds true in $W_{0}^{1, q}(\Omega)$. Let $\phi \in W_{0}^{1, s}(\Omega), \phi \geq 0$, and $u$ the solution of (1.1)-(1.3). Then:

(i) $T^{*}=\infty$.

(ii) If the Poincaré inequality holds also in $H_{0}^{1}(\Omega)$, there exists some $M(\Omega)>0$ such that for all $\mu \geq M(\Omega), u(t,$.$) is bounded and decays$ exponentially to zero in $L^{r}(\Omega)(s \leq r \leq \infty)$, as $t \rightarrow \infty$.

THEOREM 2 (unbounded solutions). - Let $q \geq p>1$ and $\mu>0$. Let $\Omega$ be a uniformly regular domain of class $C^{2}$ in $\mathbb{R}^{N}$, with inradius $\rho(\Omega)=\infty$. Then there exists $\phi \in W_{0}^{1, s}(\Omega), \phi \geq 0$, such that the solution $u$ of $(1.1)-(1.3)$ satisfies eilher

$$
T^{*}<\infty \text { and } \limsup _{t \rightarrow T^{*}}\|u(t)\|_{L^{\infty}(\Omega)}=\infty
$$

or

$$
T^{*}=\infty \text { and } \lim _{t \rightarrow \infty}\|u(\ell)\|_{L^{\infty}(\Omega)}=\infty .
$$

COROLLARY $3 *$. Let $N \leq 2, q \geq p>1$, and let $\Omega$ be a uniformly regular domain of class $C^{2}$ in $\mathbb{R}^{N}$. If $N=2$, assume that $\Omega$ is finitely connected and $q \geq 2$. Then the following are equivalent:

(i) There exists some $M(\Omega)>0$ such that for all $\mu \geq M(\Omega)$ and $\phi \in W_{0}^{1, s}(\Omega), \phi \geq 0$, the solution of (1.1)-(1.3) is global and uniformly bounded,

(ii) the Poincaré inequality holds in $H_{0}^{1}(\Omega)$,

(iii) $\rho(\Omega)<\infty$.

* Note added in proof: One can show that Corollary 3 is actually true for all $N \geq 1$ and without the assumptions $\Omega$ finitely connected and $q \geq 2$; see [S3]. 
We will also derive various results concerning global existence, boundedness or unboundedness of solutions for equation (1.1):

- If $\Omega$ contains a cone (in particular $\mathbb{R}^{N}$ ), and $q \geq p$, there exists unbounded global solutions.

- Suppose $q \geq p$. If $\Omega=\mathbb{R}^{N}$, then some solutions blow-up in infinite time at every point of $\mathbb{R}^{N}$. On the contrary, if $\Omega \neq \mathbb{R}^{N}$, blow-up (in finite or infinite time) can occur only at infinity.

- In any domain $\Omega$ (in particular in $\mathbb{R}^{N}$ ), for $q \geq p$, the solution exists globally whenever $\phi$ has exponential decay in at least one direction.

- When $q \geq p$, if the restriction of $\phi$ to some cone contained in $\Omega$ has a slow enough decay at infinity, the solution blows up in finite or infinite time (this is known to happen in finite time when $1<q<p$, see $[\mathrm{SW}])$.

- When $\Omega$ is contained in a strip, the solutions are global and uniformly bounded for all $\phi$ if $q \geq p$, and for small $\phi$ if $1<q<p$ (with $\mu$ large if $1<q<2 p /(p+1)$ ).

- We provide a qualitative description of the blow-up set when $q \geq p$, for any unbounded solution (global or not); roughly speaking, the blow-up cannot be local.

Last, we will show that our method for proving the existence of unbounded solutions also applies to convection-reaction-diffusion equations of generalized Burgers' type:

$$
u_{t}-\Delta u=u^{p}+a \cdot \nabla\left(u^{q}\right),
$$

which have been previously considered in [LPSS], [AE] and [Fr2]. This partially anwers a question raised in [AE].

The paper is organized as follows. Theorem 1 and the other boundedness properties are proved in Section 2. In Section 3, we prove Theorem 2, the other facts concerning unbounded solutions, and present the extensions to equation (1.6).

In order to clearly present the fundamental ideas of our proofs, we have relegated a number of unpleasant technicalities to three appendices. We have chosen an approach based on Lebesgue and Sobolev spaces, rather than $C^{\alpha}$ spaces, since the basic a priori estimate we obtain is in $L^{r}(\Omega)$. In Appendix A, we specify the properties of uniformly regular domains which we need, and give a detailed account of the local theory for equation (1.1) - via the corresponding integral equation - in $W_{0}^{1, s}(\Omega)$, where the domain $\Omega$ is not necessarily bounded, and where $s<\infty$ is sufficiently large. Also, we show that if the initial datum $\phi$ is sufficiently regular, then the resulting "mild" solution is a classical solution of (1.1)-(1.3). Appendix B establishes 
weak comparison principles necessary to our arguments. Indeed, many of our proofs use subsolutions and supersolutions, which we need to compare with $W_{0}^{1, s}$ solutions of the integral equation. Finally, in Appendix $\mathrm{C}$, we show that gradient blow-up does not occur, i.e. that an a priori bound in $L^{r}(\Omega)$ prevents blow-up in $W_{0}^{1, s}(\Omega)$.

Throughout Sections 2 and 3, we freely use results proved in the appendices, with appropriate citation.

\section{GLOBAL EXISTENCE AND BOUNDEDNESS}

The key ingredient in our global existence results will be an $L^{r}$ estimate that proves that the $L^{r}$ norm of $u(t)$ cannot blow-up in finite time (with $r$ finite in Theorem 2 or $r=\infty$ in the results of Section 3). However, since the local existence space is $W_{0}^{1, s}(\Omega)$, we will have to make sure that nonexplosion in $L^{r}$ norm prevents explosion in $W_{0}^{1, s}$ norm. This is the purpose of the following proposition, which is proved in Appendix C.

Proposition 2.1. - Let $\Omega$ be any regular domain in $\mathbb{R}^{N}, p, q>1$ and $\mu>0$. Let $\phi \in W_{0}^{1, s}(\Omega), \phi \geq 0$, and $u$ the solution of (1.1)-(1.3). Assume that $T^{*}<\infty$. Then $\lim \sup _{t \rightarrow T^{*}}\|u(t)\|_{r}=\infty$ for all $r$ such that $s \leq r \leq \infty$ and $r>N p / 2$.

An analogous result has been proved by Quittner [Q2, Theorem 5.1 (i)] in the bounded domain framework and working with $L^{\infty}$ and $W^{1, \infty}$ norms. His proof relies on the Bernstein device, and on a careful analysis of the tangential derivatives near the boundary, which makes essential use of the negative sign in front of the gradient term. The central part of the argument can be essentially transposed to the present context (see Lemma C2). However, some care is needed when working in an unbounded domain and with the $W_{0}^{1, s}$ theory. In particular, as we start from $L^{r}$ estimates and as $\Omega$ is now unbounded, the passage from $L^{r}$ to $L^{\infty}$ (see Lemma $\mathrm{Cl}$ ) and from $W^{1, \infty}$ to $W^{1, s}$ (see Lemma A2) has to be made clear.

Assuming this proposition, we are able to prove Theorem 1.

Proof of Theorem 1. - Let us first assume that $\phi \in C_{C}^{3}(\Omega)$, so that, by Propositions A3 and A4, for any finite $r \geq s$,

$u \in C^{1}\left(\left[0, T^{*}\right), L^{r}(\Omega)\right)$ and $\left.\forall t \in\left[0, T^{*}\right), u(t) \in W_{0}^{1, r}(\Omega)\right) \cap W^{2, r}(\Omega)$, 
and $u$ verifies (1.1)-(1.3). We multiply equation (1.1) by $u^{r-1}$ and integrate over $\Omega$, which yields, for $t \in\left[0, T^{*}\right)$,

$$
\frac{d}{d t} \int \frac{u^{r}}{r}=\int u^{r-1} \Delta u+\int u^{p+r-1}-\mu \int u^{r-1}|\nabla u|^{q} .
$$

Hence, by Green's formula

$$
\begin{aligned}
\frac{d}{d t} \int \frac{u^{r}}{r}= & -\int(r-1) u^{r-2}|\nabla u|^{2}+\int u^{p+r-1}-\mu \int u^{r-1}|\nabla u|^{q} \\
= & -\int(r-1) u^{r-2}|\nabla u|^{2}+\int u^{p+r-1}-\mu\left(\frac{q}{q+r-1}\right)^{q} \\
& \int\left|\nabla\left(u^{\frac{q+r-1}{q}}\right)\right|^{q} .
\end{aligned}
$$

Applying the Poincaré inequality in $W_{0}^{1, q}(\Omega)$, we get

$$
\begin{aligned}
\frac{1}{r} \frac{d}{d t} \int u^{r} \leq & -\int(r-1) u^{r-2}|\nabla u|^{2} \\
& +\int u^{p+r-1}-\mu C_{q}(\Omega)\left(\frac{q}{q+r-1}\right)^{q} \int u^{q+r-1}
\end{aligned}
$$

In the case $1<p<q, \mu>0$, the inequality

$$
x^{p+r-1} \leq \epsilon x^{q+r-1}+C(p, q) \epsilon^{-(p-1) /(q-p)} x^{r}, \quad x \geq 0, \epsilon>0
$$

now implies that

$$
\frac{d}{d t} \int \frac{u^{r}}{r} \leq C \int u^{r}
$$

for some $C=C(\Omega, p, q, r)>0$, and the same inequality follows from (2.2) when $q=p$ and $\mu \geq C_{q}(\Omega)^{-1}\left(\frac{q+r-1}{q}\right)^{q}$. Integrating, one then immediately obtains

$$
\int u^{r}(t) \leq\left(\int u^{r}(0)\right) \exp (C t), t \in\left[0, T^{*}\right) .
$$

In particular, the estimate 2.4 implies that $T^{*}=\infty$ by Proposition 2.1 . Suppose now that the Poincaré inequality also holds in $H_{0}^{1}(\Omega)$. Thus 2.2 implies that

$$
\begin{aligned}
\frac{1}{r} \frac{d}{d t} \int u^{r} & \leq-\frac{4(r-1)}{r^{2}} \int\left|\nabla\left(u^{r / 2}\right)\right|^{2} \\
& +\int u^{p+r-1}-\mu C_{q}(\Omega)\left(\frac{q}{q+r-1}\right)^{q} \int u^{q+r-1} \\
& \leq-\frac{4(r-1)}{r^{2}} C_{2}(\Omega) \int u^{r}+\int u^{p+r-1} \\
& -\mu C_{q}(\Omega)\left(\frac{q}{q+r-1}\right)^{q} \int u^{q+r-1}
\end{aligned}
$$


When $1<p \leq q$ and $\mu>M(\Omega, r)$ large enough, (2.5) and (2.3) yield

$$
\frac{1}{r} \frac{d}{d t} \int u^{r} \leq-K \int u^{r}
$$

for some $K=K(\Omega, r)>0$. Hence,

$$
\int u^{r}(t) \leq\left(\int u^{r}(0)\right) \exp (-K t), t \in\left[0, T^{*}\right) .
$$

A standard approximation argument (using continuous dependence in $W_{0}^{1, s}(\Omega)$ - Proposition A1) and the embedding $W_{0}^{1, s}(\Omega) \subset L^{r}(\Omega)$, since $r \geq s>N$, show that (2.4) and (2.6) are true for all initial data $\phi \in W_{0}^{1, s}(\Omega)$.

In order to prove exponential decay in $L^{\infty}$, we write the variation of constants formula between $t$ and $t+1$ :

$$
u(t+1)=e^{\Delta} u(t)+\int_{0}^{1} e^{(1-\tau) \Delta}\left(u^{p}(t+\tau)-\mu|\nabla u(t+\tau)|^{q}\right) d \tau .
$$

By the nonnegativeness of $u$ (Lemma B1), the $L^{\infty}$ estimate for the heat kernel and inequality (2.6), we obtain

$$
\begin{aligned}
\|u(t+1)\|_{\infty} & \leq C\|u(t)\|_{s}+\int_{0}^{1} C(1-\tau)^{-\theta}\|u(t+\tau)\|_{s}^{p} d \tau \\
& \leq C\left(\|u(t)\|_{s}+\sup _{0 \leq \tau \leq 1}\|u(t+\tau)\|_{s}^{p} d \tau\right) \\
& \leq C \exp \left[-C^{\prime} t\right]
\end{aligned}
$$

where $0<\theta<1$ (since $s>N p$ - see (A5)). Interpolating between $L^{s}$ and $L^{\infty}$, this proves in addition that $K$ and $M$ can actually be chosen uniformly with respect to $r \in[s, \infty]$. This completes the proof.

Remark 2.1. - When $1<p \leq q<2$, the conclusions of Theorem 1 remain valid for any $\phi \in L^{r}(\Omega)$ with $r$ large enough. Indeed the problem is well-posed in this space (as indicated in [AW, p. 16]) and it is thus sufficient to check that the $L^{r}$ norm of the solution cannot blow-up in finite time.

Remark 2.2. - a) The largeness assumption on $\mu$ for (exponential) decay to 0 cannot be relaxed in general. Indeed, if $q>2 p /(p+1)$ and $(N-2) p<(N+2)$, there exist positive stationary solutions when $\Omega$ is a ball of large radius, or equivalently for small $\mu$ when the radius is fixed (see [CW, Corollary 5.4 (ii)]). 
b) Convergence to 0 (in $W_{0}^{1, s}$ ) for $\mu$ large is proved in [F, Remark p. 800$]$ in the special case when $\Omega$ is a ball, $1<p \leq q \leq 2$. However, no estimate on the rate of convergence is provided since the result stems from a precompactness property of the trajectory and the fact (see [CW, Corollary 5.4 (i)]) that no nontrivial nonnegative stationary solution exists.

We now return to the case of a general unbounded domain with $q \geq p$. The results of Section 3 will prove that there can exist global unbounded solutions, in particular in $\mathbb{R}^{N}$. While the question of whether there exist (finite time) blow-up solutions when $q \geq p$ remains open, we are able to exclude this possibility for a certain class of initial data, specifically for $\phi$ having exponential decay in at least one direction.

THEOREM 2.2. - Let $\Omega$ be any regular domain in $\mathbb{R}^{N}, q \geq p>1, \mu>0$. Iet $\epsilon>0$, with $\epsilon \geq \mu^{-1 / p}$ if $q=p$. Assume that $\phi \in W_{0}^{1, s}(\Omega)$ satisfies the following exponential decay condition:

$$
\exists C>0, \exists a \in \mathbb{R}^{N},\|a\|=1, \forall x \in \Omega, 0 \leq \phi(x) \leq C e^{-\epsilon|x \cdot a|} .
$$

Then $T^{*}=\infty$. Moreover, there exists $\alpha>0$ such that

$$
0 \leq u(t, x) \leq C e^{\alpha t} e^{-\epsilon|x \cdot a|}
$$

for all $t>0$ and $x \in \Omega$.

Proof of Theorem 2.2. - Without loss of generality, we may assume that $a$ is the unit vector in the $x_{1}$-direction. We claim that, for the right choice of $\alpha$, the functions

$$
v_{ \pm}(t, x)=C \exp \left(\alpha t \pm \epsilon x_{1}\right)
$$

are (traveling-wave) supersolutions. If $q>p$ and $\beta>0$ or if $q=p$ and $0<\beta \leq 1$, we have the elementary inequality

$$
x^{q} \geq \beta^{(q-p) /(q-1)} x^{p}-\beta x, x \geq 0 .
$$

Therefore,

$$
\begin{aligned}
\partial_{t} v_{ \pm} & -\Delta v_{ \pm}+\mu\left|\nabla v_{ \pm}\right|^{q}-v_{ \pm}^{p} \\
& \geq \partial_{t} v_{ \pm}-\Delta v_{ \pm}+\mu \beta^{(q-p) /(q-1)}\left|\nabla v_{ \pm}\right|^{p}-\mu \beta\left|\nabla v_{ \pm}\right|-v_{ \pm}^{p} \\
& =C \exp \left(\alpha t \pm \epsilon x_{1}\right)\left(\alpha-\epsilon^{2}-\mu \beta \epsilon\right) \\
& +C^{p} \exp \left[p\left(\alpha t \pm \epsilon x_{1}\right)\right]\left(\mu \beta^{(q-p) /(q-1)} \epsilon^{p}-1\right) .
\end{aligned}
$$

Vol. $16, n^{\circ} 3-1999$. 
It thus suffices to choose $\beta=\left(\mu \epsilon^{p}\right)^{-(q-p) /(q-1)}$ and $\alpha=\epsilon^{2}+\mu \beta \epsilon$, if $q>p$, or $\beta=1$, and $\alpha=\epsilon^{2}+\mu \epsilon$ if $q=p$ and $\epsilon \geq \mu^{-1 / p}$. Then we get, thanks to the comparison principle (Lemma B1),

$$
0 \leq u(t, x) \leq v_{ \pm}(t, x), t \geq 0, x \in \mathbb{R}
$$

and so

$$
0 \leq u(t, x) \leq C \exp \left(\alpha t-\epsilon\left|x_{1}\right|\right) \quad t \geq 0, x \in \mathbb{R}
$$

In particular, Proposition 2.1 implies that $T^{*}=\infty$.

Remark 2.3. - The result of Theorem 2.2 shows that the conjecture (1.4) holds true for any $N \geq 1, \mu>0$ and any domain $\Omega$, as long as one considers only the class of initial data having exponential decay in at least one direction (and in particular in the class of data with compact support). Indeed, Theorem A provides blow-up data with compact support for $q<p$.

For the next result, we assume that the domain $\Omega$ is contained in an infinite strip (this is a special case of Theorem 1, since the Poincaré inequality is then of course valid). In this particular case, it is possible to improve the result of Theorem 1 (i), by proving that if $q \geq p$, then the solutions in fact remain bounded in $L^{\infty}$ for any $\mu>0$. In the case $1<q<p$, although blow-up solutions always exist by virtue of Theorem A, we can however prove boundedness and global existence for all small data in $L^{\infty}$ norm. The various cases are collected in the following result.

Proposition 2.3. - Let $\Omega$ be a regular domain of $\mathbb{R}^{N}$, contained in a strip, and $\phi \in W_{0}^{1, s}(\Omega), \phi \geq 0$. Assume that one the following conditions is met:

(i) $1<p \leq q, \mu>0$,

(ii) $2 p /(p+1) \leq q<p, \mu \geq \mu_{0},\|\phi\|_{\infty} \leq L(\mu)$,

(iii) $1<q<2 p /(p+1), \mu>0$, $\|\phi\|_{\infty} \leq L(\mu)$.

(Here $\mu_{0}=\mu_{0}(p, q, \Omega)$ and $L(\mu)=L(p, q, \Omega, \mu)$ are positive constants. Moreover $L(\mu)$ can be chosen so that $\lim _{\mu \rightarrow \infty} L(p, q, \Omega, \mu)=\infty$ when $p$, $q$ and $\Omega$ are fixed.). Then the solution of (1.1)-(1.3) is global and bounded in $L^{\infty}$. Moreover the bound on $u$ depends only on $\|\phi\|_{\infty}$.

Remark 2.4. - This proposition yields a slightly stronger version of conjecture (1.4) than Theorem 2, in that we do not need to assume $\mu$ large when $q=p$.

Remark 2.5. - The cases (ii)-(iii) of Proposition 2.3 can be enlightened in the following way. Consider a domain $\Omega$ containing balls of arbitrarily large radius (hence not included in a strip), e.g. $\Omega=\mathbb{R}^{N}$. By using standard 
rescaling and maximum principle arguments, along with Theorem $\mathrm{A}$, one can construct blow-up initial data of arbitrarily small $L^{\infty}$ norm. When $2 p /(p+1) \leq q<p$, this is possible for any fixed $\mu>0$. When $q<2 p /(p+1)$, this corresponds to $\mu$ tending to 0 . Moreover, when $q=2 p /(p+1), p$ is close to 1 , and $\mu$ is small enough, it is possible to construct self-similar solutions to (1.1) on $\mathbb{R}^{N}$ that blow-up exactly in one point, as $t$ increases to 1 , and tend uniformly to 0 as $t$ goes to $-\infty$ (see [STW]). This also provides blow-up data of arbitrarily small $L^{\infty}$ norm.

The methods we use for proving Proposition 2.3 are completely different from those in Theorem 1. We extend an idea of Fila $[F]$ based on supersolutions. Proposition 2.3 in fact improves the corresponding result $[\mathrm{F}$, Theorem 3] which was restricted to $q \geq 2 p /(p+1), \Omega$ bounded. Note that the point of view of $[\mathrm{F}]$ is rather to consider the value of $\mu$ as depending on $\|\phi\|_{\infty}$.

Proof of Proposition 2.3. - Without loss of generality, we can assume $\Omega \subset(0, a) \times \mathbb{R}^{N-1}$. We are seeking a supersolution of the form $v(t, x)=C e^{\alpha x_{1}}$, where $x=\left(x_{1}, \cdots, x_{N}\right)$, with $C \geq\|\phi\|_{\infty}$ and $\alpha>0$ to be determined. (Proposition 2.1 will of course imply that $T^{*}=\infty$.) The condition to ensure is thus

$$
-\alpha^{2}+\mu \alpha^{q} C^{q-1} e^{\alpha(q-1) x_{1}}-C^{p-1} e^{\alpha(p-1) x_{1}} \geq 0,0<x_{1}<a .
$$

This is achieved as soon as

$$
\mu C^{q-1} \geq 2 \alpha^{2-q}
$$

and

$$
\begin{cases}\mu \alpha^{q} C^{q-p} \geq 2 & \text { if } q \geq p, \\ \mu \alpha^{q} C^{q-p} \geq 2 e^{\alpha(p-q) a} & \text { if } q<p .\end{cases}
$$

In the case $q \geq p$, it suffices to choose

$$
\alpha=(2 / \mu)^{1 / q} \quad \text { and } \quad C \geq \max \left(1,\|\phi\|_{\infty},\left(2 \alpha^{2-q} / \mu\right)^{1 /(q-1)}\right) .
$$

In the case $q<p,(2.8)$ and (2.9) are satisfied if $\alpha$ and $C$ verify

$$
\left(\frac{2}{\mu}\right)^{1 /(q-1)} \alpha^{(2-q) /(q-1)} \leq C \leq\left(\frac{\mu}{2}\right)^{1 /(p-q)} \alpha^{q /(p-q)} e^{-\alpha a}
$$

Such $\alpha$ and $C$ exist if

$$
\left(\frac{\mu}{2}\right)^{\frac{1}{p-q}+\frac{1}{q-1}}>\sigma=\inf _{\alpha>0} \alpha^{\frac{2-q}{q-1}-\frac{q}{p-q}} e^{\alpha a} .
$$

Vol. 16, n 3-1999. 
If $q<2 p /(p+1)$, then $(2-q) /(q-1)>q /(p-q)$ and $\sigma=0$, so that suitable $\alpha$ and $C$ can be found for any $\mu>0$. If $2 p /(p+1) \leq q<p$, then $\sigma>0$, and suitable $\alpha$ and $C$ can be found for large enough $\mu$. In both cases, we take $L(\mu)=C$.

Finally, note that for $1<q<p$ and $\mu$ large enough, one can always take $\alpha=1$ and $C=(\mu / 2)^{1 /(p-q)} e^{-a}$. Therefore, $L(\mu) \rightarrow \infty$, as $\mu \rightarrow \infty$.

\section{UNBOUNDED SOLUTIONS}

The proofs of Theorem 2 and of some of the other results in this section depend in a fundamental way on the following lemma.

LEMMA 3.1. - Iet $p>1, q>2 p /(p+1)$ and $\mu \geq 0$. There exists $\eta, \epsilon$, $R>0$ and a (radial) function $v \geq 0$, of class $C^{2}$ on $\mathbb{R}^{+} \times \mathbb{R}^{N}$, satisfying:

(i) $P_{\mu} v=v_{t}-\Delta v-v^{p}+\mu|\nabla v|^{q} \leq 0$ on $\mathbb{R}^{+} \times \mathbb{R}^{N}$,

(ii) $\forall t \geq 0, \operatorname{Supp}(v(t)) \subset B(0, R+\eta t)$,

(iii) $\forall t \geq 0,\|v(t)\|_{\infty}=v(t, 0) \geq \epsilon t$,

(iv) $\forall x \in \mathbb{R}^{N}, \lim _{t \rightarrow \infty} \uparrow v(t, x)=+\infty$,

(v) $\|\nabla v\|_{L^{\infty}\left(\mathbb{R}^{+} \times \mathbb{R}^{N}\right)} \leq 1$ and $\forall t \geq 0, \forall x \in \mathbb{R}^{N}, v_{t}(t, x) \geq 0$.

Intuitively, the idea is to seek an unbounded global subsolution, whose gradient remains uniformly bounded, so that the damping effect of the gradient term can never become too important even for large $q$. This subsolution will take the form of a spherical "expanding wave", which propagates radially away from the origin with an increasing maximum at 0 .

Proof of Lemma 3.1. - We need two auxiliary functions. Let us first define a function $f: \mathbb{R} \rightarrow \mathbb{R}$, of class $C^{2}$, by

$$
f(s)= \begin{cases}0, & s \leq 0, \\ 4 s^{3}(1-s), & 0 \leq s \leq 1 / 2 \\ s-1 / 4, & s \geq 1 / 2\end{cases}
$$

It is easily seen that $f$ satisfies, for some $\epsilon>0$,

$$
\begin{gathered}
0 \leq f^{\prime} \leq 1, f^{\prime \prime} \geq 0, \quad s \in \mathbb{R} \\
f^{\prime \prime}+f^{p} \geq 3 \epsilon f^{\prime}, s \leq 1 / 2 \quad \text { and } \quad f^{p} \geq 3 \epsilon f^{\prime}, s \geq 1 / 2 .
\end{gathered}
$$


Next, we define $\beta: \mathbb{R}^{+} \rightarrow \mathbb{R}$, as

$$
\beta(s)= \begin{cases}\frac{\epsilon}{N} \frac{M^{2} s+(M-s)^{3} / 3}{2 M}, & 0 \leq s \leq M, \\ s, & s>M,\end{cases}
$$

with $M=2 N / \epsilon$. The function $\beta$ is of class $C^{2}$ on $\mathbb{R}^{+}$, with the following properties:

$$
\begin{gathered}
0 \leq \beta(s) \leq M, 0 \leq s \leq M \\
s \leq \beta(s), 0 \leq \beta^{\prime} \leq 1,0 \leq \beta^{\prime \prime} \leq \epsilon / N, \quad s \in \mathbb{R}^{+}, \\
\beta(0)-M / 3, \beta^{\prime}(0)=0 .
\end{gathered}
$$

Now we set

$$
U(t, x)=f\left(M+\frac{1}{2}+\epsilon t-\beta(|x|)\right), t \geq 0, x \in \mathbb{R}^{N},
$$

which is of class $C^{2}$ on $\mathbb{R}^{+} \times \mathbb{R}^{N}$. We compute (omitting the argument in $f, f^{\prime}, f^{\prime \prime}$ for simplicity):

$$
\begin{gathered}
\nabla U=-\frac{x}{|x|} \beta^{\prime}(|x|) f^{\prime} \quad(0 \text { if } x=0), \\
\Delta U=\beta^{\prime 2}(|x|) f^{\prime \prime}-f^{\prime} \Delta \beta(|x|), \\
\Delta \beta(|x|)=\beta^{\prime \prime}(|x|)+\frac{N-1}{|x|} \beta^{\prime}(|x|) \leq N \sup \beta^{\prime \prime} \leq \epsilon .
\end{gathered}
$$

First taking $\mu=\epsilon$ in equation (1.1), we have

$$
\begin{aligned}
P_{\epsilon} U & =\epsilon f^{\prime}-\beta^{2}(|x|) f^{\prime \prime}+\Delta \beta(|x|) f^{\prime}-f^{p}+\epsilon\left|\beta^{\prime}(|x|) f^{\prime}\right|^{q} \\
& \leq 3 \epsilon f^{\prime}-\beta^{\prime 2}(|x|) f^{\prime \prime}-f^{p} .
\end{aligned}
$$

If $s=1 / 2+M+\epsilon t-\beta(|x|) \geq 1 / 2$, then $f^{p} \geq 3 \epsilon f^{\prime}$ hence $P_{\epsilon} U(t, x) \leq 0$. On the other hand, if $s \leq 1 / 2$, then $\beta(|x|) \geq M+\epsilon t \geq M$. Hence $\beta^{\prime}(|x|)=1$ and $P_{\epsilon} U(t, x) \leq 3 \epsilon f^{\prime}-f^{\prime \prime}-f^{p} \leq 0$. Now, for a general $\mu>0$, replacing $U$ by

$$
U_{\alpha}(t, x)=\alpha^{2 /(p-1)} U\left(\alpha^{2} t, \alpha x\right)
$$

we get

$$
\begin{aligned}
\Gamma_{\mu} U_{\alpha} & -\alpha^{2 p /(p-1)}\left[U_{t}-\Delta U-U^{p}+\mu \alpha^{(q(p+1)-2 p) /(p-1)}|\nabla U|^{q}\right]\left(\alpha^{2} t, \alpha x\right) \\
& \leq \alpha^{2 p /(p-1)}\left[P_{\epsilon} U\right]\left(\alpha^{2} t, \alpha x\right) \leq 0
\end{aligned}
$$

Vol. 16, $\mathrm{n}^{\circ} 3-1999$. 
for $\alpha>0$ sufficiently small since $q>2 p /(p+1)$, which proves (i) with $v=U_{\alpha}$.

Finally, (ii)-(v) are straightforward consequences of the definition of $f$ (take $R=(M+1 / 2) / \alpha$ and $\eta=\epsilon(\chi)$.

Proof of Theorem 2. - Let us fix a sequence of positive reals $R_{n} \rightarrow \infty$. From the hypotheses, there is a sequence of disjoint balls $B_{n}=B\left(x_{n}, R_{n}^{\prime}\right) \subset \Omega$, with $R_{n}^{\prime}>R_{n}$. We are going to construct a suitable subsolution $w=w(t, x)$ of (1.1)-(1.3) on $\Omega$ by taking advantage of the rescaling properties of the equation. With $v$ as in Lemma 3.1, we set:

$$
w_{n}(t, x)=\frac{1}{n^{2 /(p-1)}} v\left(\frac{\gamma_{n}(t)}{n^{2}}, \frac{x-x_{n}}{n}\right), t \geq 0, x \in \mathbb{R}^{N}, n \in \mathbb{N}^{*},
$$

with $\gamma_{n}(t)=M_{n} t /\left(M_{n}+t\right)$, where the constants $M_{n}>0$ shall bc adjusted later. By (ii)-(iii) in Lemma 3.1, we have:

$$
\begin{gathered}
\operatorname{Supp}\left(w_{n}(t, .)\right) \subset B\left(x_{n}, n\left(R+\eta M_{n} / n^{2}\right)\right), \forall t \geq 0, \\
\left\|w_{n}(t)\right\|_{\infty} \geq \frac{\epsilon \gamma_{n}(t)}{n^{2 p /(p-1)}} \rightarrow \frac{\epsilon M_{n}}{n^{2 p /(p-1)}} \text { as } t \rightarrow \infty
\end{gathered}
$$

From (i), it follows that

$$
\begin{aligned}
P w_{n}= & n^{-2 p /(p-1)}\left[\gamma_{n}^{\prime}(t) v_{t}-\Delta v-v^{p}+\mu n^{(2 p-q(p+1)) /(p-1)}|\nabla v|^{q}\right] \\
& \left(\frac{\gamma_{n}(t)}{n^{2}}, \frac{x-x_{n}}{n}\right) \\
\leq & n^{-2 p /(p-1)}\left[v_{t}-\Delta v-v^{p}+\mu|\nabla v|^{q}\right]\left(\frac{\gamma_{n}(t)}{n^{2}}, \frac{x-x_{n}}{n}\right) \\
\leq & 0,(t, x) \in \mathbb{R}^{+} \times \mathbb{R}^{N},
\end{aligned}
$$

where we have used the fact that $q \geq p>2 p /(p+1), v_{t} \geq 0$ and $\gamma_{n}^{\prime}(t)=M_{n}^{2} /\left(M_{n}+t\right)^{2} \leq 1$. We now choose

$$
M_{n}=n^{1+2 p /(p-1)} \quad \text { and } \quad R_{n}=n\left(R+\eta M_{n} / n^{2}\right)
$$

and define the function $w$ as:

$$
w=\sum_{n \geq 1} w_{n}
$$

Note that each $w_{n}$ is supported on $B_{n}$ and that the $B_{n}$ are disjoint. By Lemma 3.1 , it is clear that $w$ is $C^{2}$ on $\mathbb{R}^{+} \times \mathbb{R}^{N}$, and hence is a classical 
subsolution of (1.1)-(1.2). Moreover, by the choice of $\gamma_{n}, w$ is bounded on $[0, T] \times \mathbb{R}^{N}$ for each $T>0$. On the other hand, since

$\left\|w_{n}(0)\right\|_{\infty} \leq \frac{1}{n^{2 /(p-1)}}\|v(0)\|_{\infty}$ and $\left\|\nabla w_{n}(0)\right\|_{\infty} \leq \frac{1}{n^{(p+1) /(p-1)}}\|\nabla v(0)\|_{\infty}$;

it follows, by the choice of $R_{n}$, that $w(0) \in W_{0}^{1 ; s}(\Omega)$ for all large $s$. Therefore, by the comparison principle (Lemma B I), the solution of (1.1)(1.3) with initial data $w(0)$ remains above $w(t)$ as long as it exists, which, along with Proposition 2.1, completes the proof.

Proof of Corollary 3. - The equivalence of (ii) and (iii) for domains of finite connectivity in dimension 2 is proved by Osserman [O, Theorem (a), p. 546]. It is obvious in dimension $N=1$, since $\Omega$ is necessarily an interval. (Note that the implication (ii) $\Rightarrow$ (iii) is clearly true in any domain and any dimension. However, it is known that the implication (iii) $\Rightarrow$ (ii) is false in dimension $N \geq 3$ - see Hayman [H], and also Lieb [L] for further results in this direction.)*

The implication (i) $\Rightarrow$ (iii) is a consequence of Theorem 2 .

Assume (ii): Then it can be seen by standard arguments that the Poincaré inequality holds also in $W_{0}^{1, q}(\Omega)$ for $q \geq 2$. The assertion (i) then follows from Theorem 1.

Under additional assumptions on $\Omega$, one can prove that some unbounded global solutions do actually exist.

PROPOSITION 3.2. - Suppose the regular domain $\Omega$ contains a cone, $\mu \geq 0$, $q \geq p>1$. There exists some $\phi \in C^{2}(\bar{\Omega}), \phi \geq 0$, with compact support, such that the solution $u$ of (1.1)-(1.3) satisfies $T^{*}=\propto$ and

$$
\lim _{t \rightarrow \infty}\|u(t)\|_{\infty}=\infty
$$

Proof of Proposition 3.2. - To prove this, we seek an unbounded solution with compact support at $t=0$, so that Theorem 2.2 applies, and supported by $\Omega$ at all time $t \geq 0$. The idea is to consider a "traveling expanding-wave", obtained by combining a spherical expanding wave such as in Lemma 3.1 and a translation motion along the axis of the cone.

Without loss of generality, one may assume that $\Omega$ contains a cone $\Omega^{\prime}$ of vertex 0 with half-axis along the first unit vector $e_{1}$. It follows that there exists some $K>1$ such that for all $r>0, \Omega^{\prime}$ contains the ball $B\left(K r e_{1}, r\right)$.

\footnotetext{
* Note added in proof: Actually, one always has (ii) $\Leftrightarrow$ (iii) when $\Omega$ is uniformly regular: see $[\mathrm{S} 3]$.

Vol. 16, n 3-1999.
} 
Let us consider the function

$$
z(t, x)=f\left(M+\frac{1}{2}+\delta t-\beta\left(\left|x-\left(A+\frac{\epsilon}{2} t\right) e_{1}\right|\right)\right), t \geq 0, x \in \mathbb{R}^{N},
$$

with $f$ and $\beta$ as in the proof of Lemma 3.1. A slight modification of the calculation in the proof of this lemma shows that

$$
P_{\epsilon} z \leq 0 \quad \text { on } \mathbb{R}^{+} \times \mathbb{R}^{N}
$$

whenever $0<\delta \leq \epsilon / 2$ and $A \geq 0$. By the usual rescaling (see formula (3.2)), we obtain

$$
P_{\mu} z_{\alpha} \leq 0 \quad \text { on } \mathbb{R}^{+} \times \mathbb{R}^{N}
$$

for all $0<\alpha \leq \alpha_{0}$, with $\alpha_{0}$ small enough (depending on $\mu$ but not on $A, \delta$ ).

From the definition of $f$ and $\beta$, we see that

$$
\operatorname{Supp}\left(z_{\alpha}(t, .)\right) \subset B\left(\left(\frac{A}{\alpha}+\frac{\epsilon}{2} \alpha t\right) e_{1}, \frac{M+1 / 2}{\alpha}+\delta \alpha t\right) .
$$

Hence, $\operatorname{Supp}\left(z_{\alpha}(t,).\right) \subset \Omega^{\prime} \subset \Omega$, for all $t \geq 0$, as long as we assume

$$
A \geq K(M+1 / 2) \text { and } \delta \leq \epsilon /(2 K) .
$$

Let us set $\phi=z_{\alpha}(0)$. Since $\phi$ has compact support, it can be bounded from above by some function of the form $C e^{|x \cdot a|}$, so that Theorem 2.2 applies. The comparison principle (Lemma B1) and Proposition 2.1 then imply the Proposition.

Remark 3.1. - From the above proof, one can deduce that $u$ actually satisfies the estimate

$$
C_{1} t \leq\|u(t)\|_{\infty} \leq C_{2} e^{C_{3} t}: \quad t \rightarrow \infty, \quad C_{1}, C_{2}, C_{3}>0 .
$$

Our next result gives a criterion for blow-up in finite or infinite time in terms of the growth of $\phi$ as $|x| \rightarrow \infty$. In the case of equation (1.1) without gradient term (i.e. $\mu=0$ ), when $\Omega=\mathbb{R}^{N}$, a result of this type was first proved by Lee and $\mathrm{Ni}[\mathrm{LN}]$, who obtained finite time blowup for any initial data $\phi$ such that $\liminf _{|x| \rightarrow \infty}|x|^{2 /(p-1)} \phi(x)$ is large enough. A similar result was proved in [STW] for equation (1.1) in the case $q=2 p /(p+1)$ and $\mu>0$ small. The authors then extended these results to general nonlinear parabolic equations of the form

$$
u_{t}-\Delta u=F(u, \nabla u),
$$


including equation (1.1) for any $1<q<p$ and $\mu>0$ [SW, Theorem 2]. Moreover, the growth condition at $\infty$ was weakened, having only to be assumed in some smaller region, specifically a cone, so that the result remains valid in any unbounded domain $\Omega$ containing a cone. The following result is thus the analog of Theorem 2 in [SW] for equation (1.1) in the case $q \geq p$ (except that we do not know whether $T^{*}<\infty$ ).

Proposition 3.3. - Let the regular domain $\Omega$ contain a cone $\Omega^{\prime}, \mu \geq 0$, $q \geq p>1$. There exists some constant $C=C\left(\Omega^{\prime}\right)>0$ such that for all $\phi \in W_{0}^{1, s}(\Omega), \phi \geq 0$, satisfying

$$
\liminf _{|x| \rightarrow \infty, x \in \Omega^{\prime}}|x|^{2 /(p-1)} \phi(x)>C
$$

then the solution $u$ of (1.1)-(1.3) is unbounded (with $T^{*} \leq \infty$ ).

The idea of the proof is similar to that of Theorem 2 in [SW]. The main difference here is that we compare $u$ to a global unbounded subsolution, instead of a (self-similar) blowing-up subsolution. We then use the properties of the equation under rescaling and translation, to "spread" the mass of the comparison function out to infinity.

Proof of Proposition 3.3. - We compare $u$ with the function $z_{\alpha}$ given in the proof of Proposition 3.2, for some possibly smaller $\alpha$, and suitable $A$.

Let us set $C=\|z(0)\|_{\infty}((K+1)(M+1))^{2 /(p-1)}$ in formula (3.3). Since $\phi$ satisfies (3.3), there is some $B>0$ such that

$$
|x| \geq B \Longrightarrow \phi(x) \geq C|x|^{-2 /(p-1)}
$$

Take $\alpha=\min \left(\alpha_{0}, 1 / B\right)$, and $A=\max (K(M+1 / 2), M+3 / 2)$. For all $x \in \Omega$ such that $z_{\alpha}(0, x) \neq 0$, we have $\left|x-A e_{1} / \alpha\right| \leq(M+1 / 2) / \alpha$, hence

$$
B \leq \frac{1}{\alpha} \leq \frac{A-(M+1 / 2)}{\alpha} \leq|x| \leq \frac{A+(M+1 / 2)}{\alpha} \leq \frac{(K+1)(M+1)}{\alpha},
$$

so that (3.4) implies

$$
z_{\alpha}(0, x) \leq \alpha^{2 /(p-1)}\|z(0)\|_{\infty} \leq\left(\frac{(K+1)(M+1)}{|x|}\right)^{2 /(p-1)}\|z(0)\|_{\infty} \leq \phi(x) .
$$

As a consequence of the comparison principle (Lemma B1), the solution $u$ with initial data $z_{\alpha}(0)$ remains above $z_{\alpha}$ as long as it exists, and the result follows. 
Remark 3.2. - With a little more work, it should be possible to prove the results of Propositions 3.2 and 3.3 for slightly more general unbounded domains, e.g. if $\Omega$ contains a paraboloï.

In the case of $I R^{N}$, we obtain global solutions that blow-up everywhere as $t \rightarrow \infty$. As a consequence of Proposition 3.5, it will turn out that this can occur only in $R^{N}$.

Proposition 3.4. - Let $\Omega=\mathbb{R}^{N}, q \geq p>1$ and $\mu>0$. There exists some $\phi \in C^{2}\left(\mathbb{R}^{N}\right), \phi \geq 0$, with compact support, such that the solution $u$ of (1.1)-(1.3) satisfies $\bar{T}^{*}=\infty$ and

$$
\forall x \in \mathbb{R}^{N}, \lim _{t \rightarrow \infty} u(t, x)=\infty .
$$

Proof of Proposition 3.4. - Taking $\phi-v(0)$, with $v$ as in Lemma 3.1, it is an immediate consequence of this lemma and Theorem 2.2.

We now provide a qualitative description of the blow-up set of all unbounded solutions (global or not) when $q \geq p$ and $\Omega$ is any (non-Poincaré) domain. We set

$$
E=\left\{x_{0} \in \bar{\Omega} \cup\{\infty\} ; \exists x_{n} \rightarrow x_{0}, \exists t_{n} \rightarrow T^{*}: u\left(t_{n}, x_{n}\right) \rightarrow \infty\right\}
$$

The situation, which is rather unusual, is described by the following alternative.

Proposition 3.5. - Let $\Omega$ be a regular domain in $\mathbb{R}^{N}, q \geq p>1$ and $\mu>0$. Assume that $\phi \in W_{0}^{1, s}(\Omega)$ is such that either $T^{*}<\infty$ or $u$ is global unbounded.

(i) If $\Omega \neq \mathbb{R}^{N}$, then $E=\{\infty\}$.

(ii) If $\Omega=\mathbb{R}^{N}$, then either $F=\mathbb{R}^{N} \cup\{\infty\}$ or $E=\{\infty\}$.

Remark 3.3. - Propositions 3.2 and 3.4 prove that there indeed exist unbounded (global) solutions with blow-up set $E$ of each of the types described in Proposition 3.5. However, we do not know whether the case $E=\{\infty\}$ can actually occur in $\mathbb{R}^{N}$.

Proof of Proposition 3.5. - (i) Since $\Omega$ is regular, one can assume that $B\left(x_{0}, \epsilon\right) \subset \Omega^{C}$ for some $x_{0} \in \mathbb{R}^{N}$ and some $\epsilon>0$. By a calculation similar to that in the proof of Proposition 2.3 (i), one easily finds that $v(t, x)=C e^{\alpha\left|x-x_{0}\right|}$ is a smooth (unbounded) stationary supersolution on $\mathbb{R}^{+} \times \bar{\Omega}$, for suitable $\alpha>0$ and $C \geq\|\phi\|_{\infty}$. By the comparison principle (Lemma $\mathrm{B} 1$ ), the solution $u(t,$.$) must then remain locally bounded in \bar{\Omega}$ for $t \in\left[0, T^{*}\right)$, hence $E=\{\infty\}$. 
(ii) Assume that some $x_{0} \in \mathbb{R}^{N}$ is not a blowup point, i.e.

$\exists t_{0} \in\left[0, T^{*}\right), \exists \epsilon>0, M=\sup \left\{u(t, x) ; t_{0} \leq t<T^{*}, x \in B\left(x_{0}, \epsilon\right) \cap \Omega\right\}<\infty$.

The function $v$ defined in part (i) is now a (smooth) supersolution on $\mathbb{R}^{+} \times\left(R^{N} \backslash B\left(x_{0}, \epsilon\right)\right)$, provided we choose $C \geq \max \left(M,\|\phi\|_{\infty},\right)$. We conclude as above.

To conclude this section, we show that our method for proving the existence of unbounded solutions also applies to other equations, namely the convection-reaction-diffusion equations of generalized Burgers' type:

$$
Q u \equiv u_{t}-\Delta u-u^{p}-a . \nabla\left(u^{q}\right)=0, t>0, x \in \mathbb{R}^{N} .
$$

This partially answers a question raised in [AE] (see the commentary before Lemma 4.6 p. 453). However it remains an open question whether blow-up can occur in finite time when $q>p$ (as it is for equation (1.1)).

Proposition 3.6. - Let $q>p>1$ and $a \in \mathbb{R}^{N}, a \neq 0$. There exists some $\phi \in C^{2}\left(\mathbb{R}^{N}\right), \phi \geq 0$, with compact support, such that the solution $u$ of (3.5) with initial data $\phi$ satisfies

$$
\lim _{t \rightarrow T^{*}}\|u(t, .)\|_{\infty}=\infty \quad\left(T^{*} \leq \infty\right)
$$

Moreover, if $T^{*}=\infty$, then we have the estimate

$$
\|u(t, .)\|_{\infty} \geq C t^{\gamma}, \quad t \rightarrow \infty
$$

with $\gamma=\min (1,1 /(q-p))$.

Proof. - By [AE, Theorem 2.1], for any $\phi \in L^{\infty}\left(\mathbb{R}^{N}\right)$ (for instance), there exists a unique maximal solution of (3.5), classical on $\left(0, T^{*}\right) \times \mathbb{R}^{N}$, such that $u \in L^{\infty}\left((0, T) \times \mathbb{R}^{N}\right)$ for all $T \in\left(0, T^{*}\right)$, and $\lim _{t \rightarrow T^{*}}\|u(t)\|_{\infty}=\infty$ if $T^{*}<\infty$.

We constuct an unbounded subsolution. Let $V(t, x)=2 v(t, x)$, where $v$ is given by Lemma 3.1 with $q=p$ and $\mu$ to be fixed later.

First assume $p<q<p+1$, hence $p /(p+1-q) \geq p$. Using Young's inequality and $|\nabla v| \leq 1$, we get

$$
\left|a . \nabla\left(v^{q}\right)\right| \leq q|a||\nabla v| v^{q-1} \leq \epsilon v^{p}+C_{\epsilon}|\nabla v|^{\frac{p}{p+1-q}} \leq \epsilon v^{p}+C_{\epsilon}|\nabla v|^{p},
$$

for any $\epsilon>0$, with some constant $C_{\epsilon}>0$.

Next assume $q \geq p+1$ and set $m=q-p \geq 1$. By modifying the function $f$ in the proof of Lemma 3.1 in such a way that $f(s)=s^{1 / m}$ 
for $s$ large, and by taking $\alpha$ small in formula (3.1), one can prove that the result of Lemma 3.1 remains valid with the additional property that $\left|\nabla\left(v^{m}\right)\right| \in L^{\infty}\left(\mathbb{R}^{+} \times \mathbb{R}^{N}\right)$, with $\left|\nabla\left(v^{m}\right)\right|_{L^{\infty}\left(\mathbb{R}^{+} \times \mathbb{R}^{N}\right)}$ arbitrarily small, and with

$$
\|v(t, .)\|_{\infty} \geq C t^{1 / m}, \quad t \rightarrow \infty
$$

instead of (iii). Then we obtain

$$
\left|a . \nabla\left(v^{q}\right)\right|=\frac{q}{q-p} v^{p} a . \nabla\left(v^{q-p}\right) \leq C v^{p}\left|\nabla\left(v^{m}\right)\right| \leq \epsilon v^{p} .
$$

Hence, in both cases,

$$
\begin{aligned}
\frac{1}{2} Q V & =v_{t}-\Delta v-2^{p-1} v^{p}-2^{q-1} a \cdot \nabla\left(v^{q}\right) \\
& \leq v_{t}-\Delta v-\left(2^{p-1}-2^{q-1} \epsilon\right) v^{p}+2^{q-1} C_{\epsilon}|\nabla v|^{p}
\end{aligned}
$$

Choosing $\epsilon$ so small that $2^{p-1}-2^{q-1} \epsilon>1$, and $\mu \geq 2^{q-1} C_{\epsilon}$, we deduce that $Q V \leq 0$, and the result follows by choosing $\phi=V(0)$. (To justify the application of the comparison principle here, see for instance [AE, Lemma 2.2 and Remark 2.3], which apply, since $V$ is $C^{2}$ and $V(t)$ has compact support for all $t$; one can also adapt the proof of Lemma B1.)

Remark 3.4. - After reading a preliminary version of this article, $\mathrm{M}$. Escobedo [E] informed us that the methods in [AE] can be used to show that, if $q>p>1$, and under the additional assumption $p<1+2 / N$, then for all $\phi \geq 0, \phi \not \equiv 0$, the solution $u$ of (3.5) cannot be global and uniformly bounded on $\mathbb{R}^{+} \times \mathbb{R}^{N}$. In other words, either $T^{*}<\infty$, or $T^{*}=\infty$ and $\limsup \sup _{t \rightarrow \infty}|u(t)|_{\infty}=\infty$.

This remark, in turn, inspired us to refine the original version of Proposition 3.6. We point out that our method of proof is completely different from those in $[\mathrm{AE}]$ (eigenfunction methods).

\section{APPENDIX A. - LOCAL THEORY AND REGULARITY OF SOLUTIONS}

\section{A.1. Introduction and preliminary facts}

Since our main results distinguish the behavior of solutions to

$$
u_{t}-\Delta u+\mu|\nabla u|^{q}=|u|^{p-1} u
$$


depending on whether or not the Poincare inequality holds on $\Omega$, it is essential that we have a local theory of solutions of (A1) on unbounded domains with unbounded boundary.

For this purpose, in all that follows, we will assume that $\Omega$ is a uniformly regular domain of class $C^{2}$, as defined in Amann [Am, Chapter III, p. 642], who refers to Browder [B]. (It seems that this definition of uniform regularity is a bit stronger than the definition in Adams [Ad, p. 67].)

Our approach to proving existence of solutions to (Al) is based on the (formally) equivalent variation of parameters integral equation:

$$
u(t)=e^{t \Delta} \phi+\int_{0}^{t} e^{(t-\tau) \Delta} J_{1}(u(\tau)) d \tau+\int_{0}^{t} e^{(t-\tau) \Delta} J_{2}(u(\tau)) d \tau,
$$

where the mappings $J_{1}$ and $J_{2}$ are defined by

$$
\begin{gathered}
J_{1}(u)=a|\nabla u|^{q}, \\
J_{2}(u)=b|u|^{p-1} u,
\end{gathered}
$$

with $q>1, p>1$, and $a$ and $b$ arbitrary real numbers. In this article, we are concerned with the case $a>0$ (i.e. $a=1$ ) and $b=-\mu<0$. While the local theory for equation (A2) does not depend on the signs of $a$ and $b$, the results of Appendix $\mathrm{C}$ depend on a crucial way on the fact that $b \leq 0(\mu \geq 0)$.

We use the abstract theory developed earlier by the second author [W]. This approach enables us to construct a local theory for equation (A2) based essentially on properties of the linear part of the equation, i.e. the heat semigroup. It is well known that the heat semigroup $e^{t \Delta}$ is well defined on an arbitrary open set and has the following properties.

(SG1) $e^{t \Delta}$ is a $C_{0}$ semigroup on $L^{r}(\Omega), 1<r<\infty$.

(SG2) $e^{t \Delta}$ is a contraction on $L^{r}(\Omega), 1 \leq r \leq \infty$, i.e.

$$
\left\|e^{t \Delta} \phi\right\|_{r} \leq\|\phi\|_{r},
$$

for all $t \geq 0$ and all $\phi \in L^{r}(\Omega)$.

(SG3) $e^{t \Delta}$ is a bounded analytic semigroup on $L^{r}(\Omega), 1<r<\infty$. In particular, there exists $M_{r}$ such that

$$
\left\|\Delta e^{t \Delta} \phi\right\|_{r} \leq M_{r} t^{-1}\|\phi\|_{r}
$$

for all $t>0$ and all $\phi \in L^{r}(\Omega)$. 
(SG4) If $1 \leq r<s \leq \infty$ and $t>0$, then $e^{t \Delta}: L^{r}(\Omega) \rightarrow L^{s}(\Omega)$ is a bounded map and

$$
\left\|e^{t \Delta} \phi\right\|_{s} \leq(4 \pi t)^{-\alpha}\|\phi\|_{r} . \quad \text { where } \alpha=\frac{N}{2}\left(\frac{1}{r}-\frac{1}{s}\right) .
$$

See (for example) the forthcoming book by Brezis and Cazenave [BC] for detailed proofs of these facts.

We denote by $D_{r}(\Delta)$ the domain of the generator of the heat semigroup in $L^{r}(\Omega)$. The following additional properties are special cases of Theorems 13.3 and 13.4 in Amann [Am].

(SG5) $D_{r}(\Delta)$, with its graph norm, is a closed subspace of $W^{2, r}(\Omega) \cap$ $W_{0}^{1, r}(\Omega)$, with equivalent norms, for all $1<r<\infty$.

(SG6) $e^{t \Delta}$ restricts to a $C_{0}$ semigroup on $W_{0}^{1, r}(\Omega)$, for all $1<r<\infty$.

Moreover, if $N<r<\infty$, then $W^{1, r}(\Omega)$ is continuously embedded into $C_{B}(\bar{\Omega})$ (see Adams [Ad, Theorem 5.4, p. 97]), and

$$
\phi(x) \rightarrow 0, \quad \text { as }|x| \rightarrow \infty . x \in \bar{\Omega}, \quad \text { for all } \phi \in W^{1 . r}(\Omega), N<r<\infty \text {. }
$$

(This is a consequence of Theorem 3.18, p. 54, Theorem 5.4, p. 97, and paragraph 4.7, p. 67 in [Ad].) By Lemma 11.1 in Amann [Am], $\Omega$ admits a strong 2-extension operator, from which one deduces the following interpolation inequalities:

$$
\begin{gathered}
\|\nabla \phi\|_{r}^{2} \leq C_{r}\|\phi\|_{2, r}\|\phi\|_{r,}, \quad 1<r<\infty, \\
\|\nabla \phi\|_{\infty}^{2 r} \leq C_{r}\|\phi\|_{2, r}^{r+N}\|\phi\|_{r}^{r-N} . \quad N<r<\infty,
\end{gathered}
$$

valid for all $\phi \in W^{2, r}(\Omega)$. Inequality (A7) follows from [Ad, Theorem 4.7 p. 79]. Inequality (A8) is proved in [Fr1, Theorem 9.3] in the case when $\Omega$ is bounded or $\Omega=\mathbb{R}^{N}$. The strong extension property implies the inequality for an unbounded domain $\Omega$. Finally, we also have the following elliptic regularity properties (see Amann [Am, Theorem 12.1]).

(ER1) If $\phi \in W_{0}^{1, r}(\Omega)$ and $\Delta \phi \in L^{r}(\Omega)$, then $\phi \in W^{2, r}(\Omega)$, and

$$
\|\phi\|_{2, r} \leq C\left(\|\Delta \phi\|_{r}+\|\phi\|_{r}\right)
$$

(ER2) If $\phi \in W_{0}^{1, r}(\Omega)$ and $\Delta \phi \in W^{1, r}(\Omega)$ then $\phi \in W^{3, r}(\Omega)$, and

$$
\|\phi\|_{3, r} \leq C\left(\|\Delta \phi\|_{1, r}+\|\phi\|_{r}\right) .
$$




\section{A.2. Statement of the local results}

Now that the assumptions on $\Omega$ and the semigroup $e^{t \Delta}$ have been specified, we can state the results of the local theory that that will be needed in the present article. The goal is to construct a local theory on the spaces $W_{0}^{1, r}(\Omega)$, where $r<\infty$ is large. Our purpose here is not to find optimal value of $r$, but rather to choose $r$ as large as we need so that the technical arguments are as simple as possible. Thus, from now on we assume that

$$
N q<r<\infty .
$$

Proposition A1. - Let $\phi \in W_{0}^{1, r}(\Omega)$, where $r$ verifies (A9).

(i) There exists a unique maximal solution $u=u(.: \phi) \quad \epsilon$ $C\left(\left[0, T_{r}(\phi)\right), W_{0}^{1, r}(\Omega)\right.$ of the integral equation (A2). If the existence time $T_{r}(\phi)$ is finite, then $\lim _{t \rightarrow T,(\phi)}\|u(t)\|_{1, r}=\infty$.

(ii) (Continuous dependence) For all $T \in\left(0, T_{r}(\phi)\right)$, there exists some neighbourhood $V$ of $\phi$ in $W_{0}^{1, r}(\Omega)$, such that $T_{r}(\psi)>T$ for all $\psi \in V$, and such that for all $t \in(0, T]$, the map $\psi \mapsto u(t ; \psi)$ is (Lipschitz) continuous from $V$ to $W_{0}^{1, r}(\Omega)$.

The next point is to make sure that if $\phi$ belongs to $W_{0}^{1, r}(\Omega)$ for more than one value of $r$, then the corresponding solutions and their maximal existence times $T_{r}(\phi)$ do not in fact depend on $r$. We will also derive some useful estimates on the $W^{1, \infty}$ norm of the solution.

Proposition A2. - Let $\phi \in W_{0}^{1, r}(\Omega)$, where $r$ verifies (A9), and let $u(t)$ be the resulting solution of (A2) in $W_{0}^{1, r}(\Omega)$.

(i) $u(t) \in W_{0}^{1, s}(\Omega)$, for all $t \in\left(0 . T_{r}(\phi)\right)$ and all $s \in(r, \infty)$. Also, $u(t) \in W^{1, \infty}(\Omega)$ for all $t \in\left(0, T_{r}(\phi)\right)$.

(ii) Let $R>0$. There exist $T>0$ and $C>0$ such that $T_{r}(\phi)>T$ for all $\phi \in U_{(r . R)}$ (the centered ball in $W_{0}^{1, r}(\Omega)$ of radius $R$ ), and

$$
\begin{aligned}
\|u(t)-v(t)\|_{1, \infty} & \leq C t^{-N / 2 r-1 / 2}\|\phi-\psi\|_{r} \\
& +C\left(\|\phi\|_{1, r}^{q-1}+\|\phi\|_{1, r}^{p-1}+\|\psi\|_{1, r}^{q-1}+\|\psi\|_{1, r}^{p-1}\right)\|\phi-\psi\|_{1, r}
\end{aligned}
$$

for all $\phi, \psi \in U_{(r . R)}$, and all $t \in[0, T]$, where $u$ and $v$ are the corresponding solutions of (A2).

(iii) If limsup $\lim _{t \rightarrow T_{r}(\phi)}\|u(t)\|_{1, \infty}<\infty$, then $T_{r}(\phi)=\infty$.

(iv) If $\phi \in W_{0}^{1, r}(\Omega) \cap W_{0}^{1, s}(\Omega)$ with $r<s<\infty$, then $T_{r}(\phi)=T_{s}(\phi)$, and the corresponding solutions in $W_{0}^{1, s}(\Omega)$ and in $W_{0}^{1, r}(\Omega)$ coincide.

Notation. - In what follows, we designate by $T^{*}(\phi)$ the common value of all $T_{r}(\phi)$, for all $r$ such that $\phi \in W_{0}^{1, r}(\Omega)$. 
Next, we turn to regularity properties of solutions of the integral equation (A2). By standard parabolic $L^{r}$-regularity theory (see e.g. [LSU]), and Proposition A2 (i), it is easy to see that, for any $\phi \in W_{0}^{1, r}(\Omega)$, the solution of (A2) given by Proposition A2 is in fact a classical solution of (A1) (that is: $(1.1))$ in $\left(0, T^{*}(\phi)\right) \times \Omega$. However, for the developments in Appendix $\mathrm{C}$, we will require $C^{2}$ solutions up to the boundary, for smooth initial data, for instance for $\phi \in C_{c}^{3}(\Omega)$. The basic tool we use is the generator of the semi-flow induced by the integral equation. Recall that if $\phi \in W_{0}^{1, r}(\Omega)$ and if $u(t)$ is the resulting solution of (A2), then the generator $B$ is defined by

$$
B \phi=W_{0}^{1, r}(\Omega)-\lim _{t \rightarrow 0^{+}} \frac{u(t)-\phi}{t},
$$

provided this limit exists. The domain of the generator, $D_{r}(B)$, is precisely the set of $\phi \in \Omega$ for which this limit exists. From [W, Theorem 2.2], we deduce the following regularity result:

Proposition A3. - Let $\phi \in D_{r}(B)$. Then:

(i) $u(t) \in D_{r}(B)$ for all $t \in\left[0, T_{r}(\phi)\right)$,

(ii) $u \in C^{1}\left(\left[0, T_{r}(\phi)\right) ; W_{0}^{1, r}(\Omega)\right)$,

(iii) $u^{\prime}(t)=B u(t)$ for all $t \in\left[0, T_{r}(\phi)\right)$.

Our main task is therefore to identify $B$ and its domain.

Proposition A4. $-D_{r}(B)$ is the set of all $\phi \in W_{0}^{1, r}(\Omega)$ such that

$$
\Delta \phi+a|\nabla \phi|^{q}+b|\phi|^{p-1} \phi \in W_{0}^{1, r}(\Omega) .
$$

Moreover,

$$
B \phi=\Delta \phi+a|\nabla \phi|^{q}+b|\phi|^{p-1} \phi, \forall \phi \in D_{r}(B)
$$

Suppose $\phi \in D_{r}(B) \cap W_{0}^{1, r q}(\Omega)$ for some $r$ verifying condition (A9). Then each of $\Delta \phi, J_{1}(\phi)$, and $J_{2}(\phi)$ is separately in $W^{1, r}(\Omega) \subset C_{B}(\bar{\Omega})$. Moreover, the resulting solution $u$ of $(\mathrm{A} 2)$ verifies $u \in C\left([0, T(\phi)), W^{3, r}(\Omega)\right)$.

The following corollary, which is an immediate consequence of Propositions A3 and A4, will be useful in the Appendices B and C.

Corollary A5. - Suppose $\phi \in C_{c}^{3}(\Omega)$, and let $u(t)$ be the solution of (A2) with initial value $\phi$. It follows that:

(a) $\phi \in D_{r}(B)$ for all $r \in(N q, \infty)$;

(b) $u \in C^{1}\left(\left[0, T^{*}(\phi)\right) ; C_{B}(\bar{\Omega})\right)$;

(c) $u^{\prime}(t)=\Delta u(t)+a|\nabla u(t)|^{q}+b|u(t)|^{p-1} u(t)$ for all $t \in\left[0, T^{*}(\phi)\right)$; 
(d) each term $\Delta u(t),|\nabla u(t)|^{q}$, and $|u(t)|^{p-1} u(t)$ is continuous into $C_{B}(\bar{\Omega})$ for $t \in\left[0, T^{*}(\phi)\right)$. In particular, $u$ is a classical solution of (A1) on $Q=\left[0, T^{*}(\phi)\right) \times \bar{\Omega}$.

\section{A.3. Proof of the Iocal results}

We begin work by giving some additional properties concerning the heat semi-group.

LEMMA A6. - Let $1<r<\infty$. For all $t>0, e^{t \Delta}: L^{r}(\Omega) \rightarrow W_{0}^{1, r}(\Omega)$ is a bounded map. Also, there exists $C_{r}>0$ such that

$$
\left\|\nabla e^{t \Delta} \phi\right\|_{r} \leq C_{r}\left(1+t^{-1 / 2}\right)\|\phi\|_{r}
$$

for all $t>0$ and all $\phi \in L^{r}(\Omega)$.

Proof. - Since $e^{t \Delta}$ is an analytic semigroup on $L^{r}(\Omega)$, it follows that $e^{t \Delta}: L^{r}(\Omega) \rightarrow D_{r}(\Delta) \subset W_{0}^{1, r}(\Omega)$ is bounded for all $t>0$, where we consider $D_{r}(\Delta)$ with its graph norm. In particular, $e^{t \Delta} \phi \in W_{0}^{1, r}(\Omega)$ for $\phi \in L^{r}(\Omega)$ and all $t>0$. Thus, we may use the interpolation inequality (A7) and property (SG5), with $\phi$ replaced by $e^{t \Delta} \phi$. This gives

$$
\left\|\nabla e^{t \Delta} \phi\right\|_{r}^{2} \leq C_{r}\left(\left\|\Delta e^{t \Delta} \phi\right\|_{r}+\left\|e^{t \Delta} \phi\right\|_{r}\right)\left\|e^{t \Delta} \phi\right\|_{r},
$$

and the proposition now is a consequence of inequalities (A3) and (A4).

LEMMA A7. - Let $1 \leq r<s \leq \infty$. For all $t>0, e^{t \Delta}: L^{r}(\Omega) \rightarrow W_{0}^{1, s}(\Omega)$ is a bounded map lexcept if $s=\infty$, then $e^{t \Delta}: L^{r}(\Omega) \rightarrow W^{1, \infty}(\Omega)$ is a bounded map]. Also, there exists $C=C(r, s)$ such that

$$
\left\|\nabla e^{t \Delta} \phi\right\|_{s} \leq C\left(1+t^{-\alpha-1 / 2}\right)\|\phi\|_{r}, \text { where } \alpha=\frac{N}{2}\left(\frac{1}{r}-\frac{1}{s}\right),
$$

for all $t>0$ and all $\phi \in L^{r}(\Omega)$.

Proof. - If $s<\infty$, the fact that $e^{t \Delta}: L^{r}(\Omega) \rightarrow W_{0}^{1, s}(\Omega)$ is a bounded map follows from the previous proposition and property (SG4) of the heat semigroup mentioned above. Also, (A13) is an immediate consequence of (A5) and (A12).

If $s=\infty$ and $r>N$, we use the Sobolev inequality (A8) and property (SG5), with $\phi$ replaced by $e^{t \Delta} \phi$. This is permitted since $e^{t \Delta} \phi \in W^{2, r}(\Omega) \cap W_{0}^{1, r}(\Omega)$. We thereby obtain

$$
\begin{aligned}
\left\|\nabla e^{t \Delta} \phi\right\|_{\infty}^{2 r} & \leq C_{r}\left(\left\|\Delta e^{t \Delta} \phi\right\|_{r}+\left\|e^{t \Delta} \phi\right\|_{r}\right)^{r+N}\left\|e^{t \Delta} \phi\right\|_{r}^{r-N} \\
& \leq C\left(1+t^{-(r+N)}\right)\|\phi\|_{r}
\end{aligned}
$$

Vol. $16, n^{\circ} 3-1999$. 
where we also have used inequalities (A3) and (A4). This proves (A13) if $s=\infty$ and $r>N$. If $s=\infty$ and $r \leq N$, then we combine (A13) with $r>N$ and (A5) to obtain the general case. The fact that $e^{t \perp}: L^{v}(\Omega) \rightarrow W^{1} \times(\Omega)$ is bounded follows from (A13) and (A5) with $s=\infty$. This proves the proposition.

To handle the integral equation (A2) via the framework of [W], we need the following property of the mappings $J_{1}$ and $J_{2}$.

LEMmA A8. - If $q<r<\infty$, then $J_{1}: W_{0}^{1, r}(\Omega) \rightarrow L^{r / q}(\Omega)$ is Lipschitz, on bounded sets and continuously Frechet differentiable. If $N<r<\infty$, then $J_{2}: W_{0}^{1, r}(\Omega) \rightarrow L^{r}(\Omega)$ is Lipschitz on bounded sets and continuously Frechet differentiable.

Proof. - It is clear that if $r>q$, then $J_{1}: W_{0}^{1, r}(\Omega) \rightarrow L^{r / q}(\Omega)$ is Lipschitz on bounded sets, and in fact continously Frechet differentiable. If $r>p$, the same is true for $J_{2}: L^{r}(\Omega) \rightarrow L^{r / p}(\Omega)$, and therefore also for $J_{2}: W_{0}^{1, r}(\Omega) \rightarrow L^{r / p}(\Omega)$. In both cases, the Frechet derivative of $J_{2}$ at a function $\phi$ applied to another function $\psi$ is given by

$$
D J_{2}(\phi) \psi=a p \int_{\Omega}|\phi|^{p-1} \phi
$$

(To simplify the notation, we restrict ourselves to real-valued functions.) We can improve this as follows. If $r>N$, then $W_{0}^{1, r}(\Omega)$ is continuously embedded into $C_{B}(\bar{\Omega})$ (Theorem 5.4, Part III, pp. 97-98 in [Ad]). In this case, we see that if $\phi \subset W_{0}^{1, r}(\Omega)$, then $D J_{2}(\phi)$ is a bounded linear map on $L^{r}(\Omega)$, and therefore also a map $W_{0}^{1, r}(\Omega) \rightarrow L^{r}(\Omega)$. Since

$$
J_{2}(\phi+\psi)-J_{2}(\phi)=\int_{0}^{1}\left[D J_{2}(\phi+t \psi)\right] \psi d t
$$

it follows that $J_{2}: W_{0}^{1, r}(\Omega) \rightarrow L^{r}(\Omega)$ is continuously Frechet differentiable (and Lipschitz on bounded sets), for all $N<r<\infty$.

We are now ready to prove the local results for (A1) aand (A2).

Proof of Proposition A1. - For each $t>0$, we define the nonlinear map $K_{t}$ by

$$
K_{t}=e^{t \Delta} J_{1}+e^{t \Delta} J_{2}
$$

Lemmas A6, A7, A8, and property (SG4) imply that $K_{t}: W_{0}^{1, r}(\Omega) \rightarrow$ $W_{0}^{1, r}(\Omega)$ is continuously Frechet differentiable, Lipschitz on bounded sets. Furthermore, if $U_{(r, R)}$ is the centered ball in $W_{0}^{1, r}(\Omega)$ of radius $R$, then

$$
\operatorname{Lip}\left(K_{t} \mid U_{(r, R)}\right) \leq C_{r}(R)\left(1+t^{-N(q-1) / 2 r-1 / 2}\right) .
$$


(In fact, $C_{r}(R)<C_{r}\left(R^{q-1}+R^{p-1}\right)$.) Condition (A9) easily implies that $N(q-1) / 2 r+1 / 2<1$, and so the integral equation (A2) generates a semi-flow on $W_{0}^{1, r}(\Omega)$ having the properties described in Theorems $1,2.2$ and 3.1 of $[\mathrm{W}]$. Note that the integral equation (A2) can be written simply as

$$
u(t)-e^{t \Delta} \phi+\int_{0}^{t} K_{t-\tau}(u(\tau)) d \tau
$$

This proves Proposition Al

Proof of Proposition A2. - (i)-(ii) Let $r<s \leq \infty$. By Lemmas A6, $\mathrm{A} 7, \mathrm{~A} 8$, and property (SG4), we see easily that for all $t>0, K_{t}$ maps $W_{0}^{1, r}(\Omega)$ into $W_{0}^{1, s}(\Omega)$ [except if $s=\infty$, in which case $K_{t}$ maps $W_{0}^{1, r}(\Omega)$ into $W^{1, \infty}(\Omega)$ ], and that

$$
\left\|K_{t}(u)\right\|_{1 . s} \leq C(r, s)\left(1+t^{-N_{q} / 2 r-1 / 2}\right)\left(\|u\|_{1, r}^{q-1}+\|u\|_{1, r}^{p-1}\right) .
$$

Condition (A9) on $r$ implies that if $0<t<T_{r}(\phi)$, then the integral term in (A14) is a convergent Bochner integral in $W_{0}^{1, s}(\Omega)\left[W^{1, \infty}(\Omega)\right.$ in case $s=\infty$ ]. Also, Lemma A7 implies that the semigroup term is in the desired space. Moreover,

$$
\begin{aligned}
\|u(t)\|_{1, s} & \leq C(r, s)\left(1+t^{-N / 2 r-1 / 2}\right)\|\phi\|_{r} \\
& +C(r, s)\left(t+t^{1 / 2-N q / 2 r}\right) \sup _{[0 . t]}\left(\|u(\tau)\|_{1, r}^{q}+\|u(\tau)\|_{1, r}^{p}\right) .
\end{aligned}
$$

In particular, if $\phi \in W_{0}^{1, r}(\Omega) \cap W_{0}^{1, s}(\Omega)$ with $r<s<\infty$, then

$$
T_{s}(\phi) \geq T_{r}(\phi)
$$

(We emphasize that these calculations do not use the optimal powers of $t$. Condition (A9) on $r$ enables us to use the same power of $t$ for all $s$ with $r<s \leq \infty$.)

If $\phi, \psi \in W_{0}^{1, s}(\Omega)$, and if $u$ and $v$ are the corresponding solutions of (A14), a calculation similar to (A15) gives

$$
\begin{aligned}
& \|u(t)-v(t)\|_{1, s} \leq C(r, s)\left(1+t^{-N / 2 r-1 / 2}\right)\|\phi-\psi\|_{r}+C(r, s)\left(t+t^{1 / 2-N / 2 r}\right) \\
& \sup _{[0, t]}\left(\|u(\tau)\|_{1, r}^{q-1}+\|u(\tau)\|_{1, r}^{p-1}+\|v(\tau)\|_{1, r}^{q-1}+\|v(\tau)\|_{1, r}^{p-1}\right)\|u(\tau)-v(\tau)\|_{1, r} .
\end{aligned}
$$

By part (iii) of Theorem 1 in [W], given $R>0$, there exists $T>0$ such that $T_{r}(\phi)>T$ for all $\phi \in U_{(r, R)}$ and the time-t maps of the semiflow Vol. 16, n० 3-1999. 
generated by (A14) are uniformly Lipschitz on $U_{(r, R)}$ for $t \in[0, T]$. This fact combined with (A17) immediately gives (A10).

(iii) Suppose that $\limsup _{t \rightarrow T_{r}(\phi)}\|u(t)\|_{1, \infty}<\infty$. Replacing $\phi$ by $u(\epsilon)$, for some small $t>0$, we may assume that $\|u(l)\|_{1, \infty} \leq C$ for all $t \in\left[0, T_{r}(\phi)\right)$. It follows that

$$
\left\|J_{1}(u(t))\right\|_{r} \leq C\|\nabla u(t)\|_{r} \leq C\|u(t)\|_{1, r},
$$

and

$$
\| J_{2}\left(u(t)\left\|_{r} \leq C\right\| u(t) \|_{r},\right.
$$

for all $t \in\left[0, T_{r}(\phi)\right)$. The integral equation (A2) now implies that

$$
\begin{aligned}
\|u(t)\|_{1, r} & \leq C\|\phi\|_{1, r}+C \int_{0}^{t}\left[1+(t-\tau)^{-1 / 2}\right]\|u(\tau)\|_{1, r} d \tau \\
& \leq C\|\phi\|_{1, r}+C\left\{\int_{0}^{t}\left[1+(t-\tau)^{-1 / 2}\right]^{3 / 2} d \tau\right\}^{2 / 3}\left\{\int_{0}^{t}\|u(\tau)\|_{1, r}^{3} d \tau\right\}^{1 / 3},
\end{aligned}
$$

from which we see that

$$
\|u(t)\|_{1, r}^{3} \leq C\|\phi\|_{1, r}^{3}+C\left(t^{2}+t^{1 / 2}\right) \int_{0}^{t}\|u(\tau)\|_{1, r}^{3} d \tau .
$$

It now follows from Gronwall's lemma that $\|u(t)\|_{1, r}^{3}$ stays bounded on any bounded subinterval of $\left[0, T_{r}(\phi)\right)$. This proves that $T_{r}(\phi)=\infty$.

(iv) The fact that the solutions arising from $\phi$ in $W_{0}^{1, s}(\Omega)$ and in $W_{0}^{1, r}(\Omega)$ coincide on $\left[0, T_{r}(\phi)\right)$ is an immediate consequence of (A16), (i), and of the local uniqueness in $W_{0}^{1, s}(\Omega)$. In view of (A15), with $s=\infty$ and $r$ replaced by $s$, (iv) follows from (iii).

Proof of Proposition A3. - This is an immediate consequence of Theorem 2.2 in [W1], using in particular the fact that the maps $K_{t}: W_{0}^{1, r} \rightarrow W_{0}^{1, r}$ are Frcchct differentiable.

Before beginning the proof of Proposition A4, we note that, by Theorem 3.1 in [W],

$$
B \phi=W_{0}^{1, r}(\Omega)-\lim _{t \rightarrow 0^{+}}\left[\Delta e^{t \Delta} \phi+e^{t \Delta} J_{1}(\phi)+e^{t \Delta} J_{2}(\phi)\right]
$$

where $D_{r}(B)$ is precisely the set of $\phi \in W_{0}^{1, r}(\Omega)$ for which this limit exists. (There is a technical point here worth mentioning. In Theorem 3.1 in [W] there is an additional requirement that $e^{t \Delta} \phi \in D_{1, r}(\Delta)$ for all $t>0$, where $D_{1, r}(\Delta)$ is the domain of $\Delta$ as a semigroup generator in $W_{0}^{1, r}(\Omega)$. 
However, since $c^{t \Delta}$ is an analytic semigroup on $L^{r}(\Omega)$, it is also an analytic semigroup on $D_{r}(\Delta)$, with its graph norm. Thus, $e^{t \Delta} \phi \in D_{r}\left(\Delta^{2}\right)$ for all $t>0$. Since $D_{r}(\Delta)$ is continuously embedded in $W_{0}^{1, r}(\Omega)$, it follows that $D_{r}\left(\Delta^{2}\right) \subset D_{1, r}(\Delta)$. It then follows that $e^{t \Delta} \phi \in D_{1, r}(\Delta)$ for all $t>0$ and for all $\phi \in W_{0}^{1, r}(\Omega)$.)

Proof of Proposition A4. - We note first that if $\phi \in W_{0}^{1, r}(\Omega)$, where $r$ verifies (A9), then $J_{2}(\phi) \in W^{1, r}(\Omega)$. Indeed, $\nabla J_{2}(\phi)=p|\phi|^{p-1} \nabla \phi$, which is in $L^{r}(\Omega)$ since $W_{0}^{1, r}(\Omega) \subset L^{\infty}(\Omega)$. Thus $e^{t \Delta} J_{2}(\phi)$ converges to $J_{2}(\phi)$ in $W^{1, r}(\Omega)$ for all $\phi \in W_{0}^{1, r}(\Omega)$. Also, $J_{1}(\phi) \in L^{r / q}(\Omega)$, and so $e^{t \Delta} . J_{1}(\phi)$ converges to $J_{1}(\phi)$ in $L^{r / q}(\Omega)$ for all $\phi \in W_{0}^{1, r}(\Omega)$. Finally, $\Delta \phi \in W^{-1, r}(\Omega)$, and so $\Delta e^{t \Delta} \phi=e^{t \Delta} \Delta \phi$ converges in $W^{-1, r}(\Omega)$ for all $\phi \in W_{0}^{1, r}(\Omega)$. In other words, if $\phi \in W_{0}^{1, r}(\Omega)$, then $\Delta e^{t \Delta} \phi+e^{t \Delta} J_{1}(\phi)+$ $e^{t \Delta} J_{2}(\phi)$ converges as a distribution to $\Delta \phi+a|\nabla \phi|^{q}+b|\phi|^{p-1} \phi$, as $t \rightarrow 0^{+}$.

Suppose that $\phi \in D_{r}(B)$. The $W_{0}^{1, r}(\Omega)$ limit (A18) must be the same as the distribution limit. Thus, $\Delta \phi+a|\nabla \phi|^{q}+b|\phi|^{p-1} \phi \in W_{0}^{1, r}(\Omega)$ and $B \phi$ is given by (A11).

On the other hand, if $\Delta \phi+a|\nabla \phi|^{q}+b|\phi|^{p-1} \phi \in W_{0}^{1, r}(\Omega)$, then

$$
\Delta e^{t \Delta} \phi+e^{t \Delta} J_{1}(\phi)+e^{t \Delta} J_{2}(\phi)=e^{t \Delta}\left(\Delta \phi+a|\nabla \phi|^{q}+b|\phi|^{p-1} \phi\right),
$$

and so the limit (A18) indeed exists.

Suppose that $\phi \in D_{r}(B) \cap W_{0}^{1, r q}(\Omega)$. We have already seen that $J_{2}(\phi) \in W^{1, r}(\Omega)$. Since $\phi \in W_{0}^{1, r q}(\Omega)$, it follows that $J_{1}(\phi) \in L^{r}(\Omega)$. The hypothesis $\phi \in D_{r}(B)$ implies that $\Delta \phi+J_{1}(\phi)+J_{2}(\phi) \in W_{0}^{1, r}(\Omega)$, and so $\Delta \phi \in L^{r}(\Omega)$. Elliptic regularity (ER1) enables us to conclude that $\phi \in W^{2, r}(\Omega)$.

We claim that $J_{1}(\phi)$ must therefore be in $W^{1, r}(\Omega)$. To show this, we need to show that $\nabla|\nabla \phi|^{q} \in L^{r}(\Omega)$. One easily verifies that $\partial_{k}|\nabla \phi|^{q}=q|\nabla \phi|^{q-2} \nabla \phi . \nabla\left(\partial_{k} \phi\right)$. Since $\phi \in W^{2, r}(\Omega)$, each component of $\nabla \phi$ is in $W^{1, r}(\Omega)$, which is embedded in $L^{\infty}(\Omega)$. Moreover, each component of $\nabla\left(\partial_{k} \phi\right)$ is in $L^{r}(\Omega)$. This proves the claim.

Since $J_{1}(\phi), J_{2}(\phi)$, and $\Delta \phi+J_{1}(\phi)+J_{2}(\phi)$ are each separately in $W^{1, r}(\Omega)$, it follows that $\Delta \phi \in W^{1, r}(\Omega)$. The elliptic regularity condition (ER2) allows us to conclude that $\phi \in W^{3, r}(\Omega)$.

By Proposition A3, $B u(t)$ is continuous into $W^{1, r}(\Omega)$. From the above bootstrap argument, applied to $u(t)$, and the elliptic estimates given in (E1) and (E2), it follows that $u(t)$ is continuous into $W^{3, r}(\Omega)$. This completes the proof. 


\section{APPENDIX B. - COMPARISON LEMMAS}

Although such type of results are well-known, we provide here in precise form the comparison lemmas that are adapted to the present context (and in particular to the case of unbounded domains, $W_{0}^{1 . s}$ solutions, and unbounded comparison functions).

Lemma B1. - Let $\phi \in W_{0}^{1, s}(\Omega), \phi \geq 0$, and $u$ be the corresponding solution of (A2). Let $T \in\left(0, T^{*}(\phi)\right), Q=(0, T] \times \Omega$.

(i) Then $u(t, x) \geq 0$ on $\left[0, T^{*}(\phi)\right) \times \bar{\Omega}$.

(ii) Assume that $v \in C(\bar{Q}) \cap C^{1.2}(Q)$ satisfies

$$
v_{t}-\Delta v+\mu|\nabla v|^{q} \geq v^{p} \text { in } Q . \quad v \geq 0 \text { in } \bar{Q},
$$

and

$$
v(0, x) \geq \phi(x) . \text { in } \bar{\Omega}
$$

Then $v \geq u$ in $Q$.

(iii) Assume that $v \in C(\bar{Q}) \cap C^{1,2}(Q)$ satisfies

$$
\begin{gathered}
v_{t}-\Delta v+\mu|\nabla v|^{q} \leq|v|^{p-1} v \text { in } Q, \\
v(0, x) \leq \phi(x) \text { in } \bar{\Omega}, \\
v \leq 0 \text { on }(0, T] \times \partial \Omega,
\end{gathered}
$$

and

$$
\limsup _{(t, x) \in \bar{Q},|x| \rightarrow \infty} v(t, x) \leq 0
$$

Then $v \leq u$ in $Q$.

Proof of Lemma B1. - (ii) Let $\dot{\phi}_{n} \in C_{c}^{3}(\Omega)$ be a sequence that approximates $\phi$ in $W_{0}^{1, s}(\Omega)$ and $u_{n}$ the corresponding solutions. By Proposition Al (ii), each $u_{n}$ exists on $[0, T]$ for $n$ large enough. Moreover, each $u_{n}$ is a classical solution by Corollary A5, and is bounded on $\bar{Q}$ by a constant $M_{n}$. Let $w=u_{n}-v$. Since $u_{n} \in C\left([0, T], W_{0}^{1, s}(\Omega)\right)$ and $v \geq 0$, it follows from (A6) and a compactness argument that

$$
\lim _{(t, r) \in \bar{Q},|x| \rightarrow \infty} w(t, x)<0 .
$$

and we have $w(t, x) \leq 0$ on the parabolic boundary $(\{0\} \times \Omega) \cup((0, T) \times$ $\partial \Omega$ ). Therefore, either $w \leq 0$ in $Q$, or the positive supremum of $w$ in $\bar{Q}$ is attained at some point of $Q$. 
On the other hand, $w$ clearly satisfies

$$
w_{t}-\Delta w \leq p M_{n}^{p-1}|w|-\mu\left|\nabla u_{n}\right|^{q}+\mu|\nabla v|^{q}
$$

on $Q$. (Consider separately the cases $u_{n}(t, x) \leq v(t, x)$ and $0 \leq v(t, x) \leq$ $u_{n}(t, x)$.) Therefore, by the maximum principle, $w$ cannot achieve a positive maximum in $Q$ (note that $\nabla u_{n}=\nabla v$ at a positive maximum). Hence, $u_{n} \leq v$ in $Q$. The result follows by letting $n \rightarrow \infty$, and using the continuous dependence (Proposition A1) and the embedding $W_{0}^{1, s}(\Omega) \subset C_{B}(\bar{\Omega})$.

(iii) The proof is similar to that of (i), by exchanging the roles of $v$ and $u_{n}$. In particular, $z=v-u_{n}$ satisfies (B2), thanks to (B1) and (A6), and $z$ also satisfies

$$
z_{+}-\Delta z \leq p M^{p-1}|z|-\mu|\nabla v|^{q}+\mu\left|\nabla u_{n}\right|^{q} .
$$

with $M=\max \left(M_{n}, M^{\prime}\right)$ and $M^{\prime}=\sup v(t, x)$, which is finite by virtue of (Bl). $(t, r) \in \bar{Q}$

(i) This is a special case of (iii) with $v=0$.

The following version of the weak maximum principle will be needed in the proof of Lemma C2. It relies on the Stampacchia method. A similar result (in the case of $W_{0}^{1, s}(\Omega)$ instead of $W^{1, s}(\Omega)$ ), proved by M. Chipot, can be found in [AW, Lemma 4.2].

Lemma B2. - Let $N<r<\infty, r \geq 2$, and $T, C>0$. Assume that $z \in C\left([0, T], W^{1, r^{\prime}}(\Omega)\right), z \in C^{1}\left((0, T], L^{r}(\Omega)\right)$, and

$$
z_{t} \quad \Delta z \leq C|\nabla z|
$$

on $(0, T]$ in the $W^{-1, r}(\Omega)$ sense. Then $K=\max \left(\sup _{x \in \Omega} z(0, x), \sup _{t \in\left[0, T^{\prime}\right], x \in d \Omega}\right.$ $z(t, x)))$ is finite, and we have $z \leq K$ on $[0, T] \times \bar{\Omega}$.

Proof of Lemma B2. - Since $W^{1, s}(\Omega) \subset C_{B}(\bar{\Omega}), z$ is uniformly bounded on $[0, T] \times \bar{\Omega}$, so that, in particular, $K$ is finite. We have $(z-K)_{+} \in C\left([0, T], W_{0}^{1, r}(\Omega)\right),(z-K)_{+} \in C^{1}\left((0 . T], L^{r}(\Omega)\right)$. Morcover,

$$
\int(z(t)-K)_{+}^{r-1} z_{t}(t) d x=\frac{d}{d t} \int \frac{(z(t)-K)_{+}^{r}}{r} d x
$$

and

$$
\left\langle\Delta z(t),(z(t)-K)_{+}^{r-1}\right\rangle_{W^{-1, r}, W_{0}^{-1, r^{\prime}}}=-(r-1) \int(z(t)-K)_{+}^{r-2}|\nabla z(t)|^{2} d x
$$


Therefore

$$
\begin{aligned}
\frac{d}{d t} \int \frac{(z(t)-K)_{+}^{r}}{r} d x & \leq-(r-1) \int(z(t)-K)_{+}^{r-2}|\nabla z(t)|^{2} d x \\
& +C \int(z(t)-K)_{+}^{r-1}|\nabla z(t)| d x
\end{aligned}
$$

Writing

$(z(t)-K)_{+}^{r-1}\left|\nabla(z(t)-K)_{+}\right| \leq \epsilon(z(t)-K)_{+}^{r-2}|\nabla z(t)|^{2}+C_{\epsilon}(z(t)-K)_{+}^{r}$,

and choosing $\epsilon$ small enough, we obtain that

$$
\frac{d}{d t} \int(z(t)-K)_{+}^{r} d x \leq C^{\prime} \int(z(t)-K)_{+}^{r} d x
$$

and the results follows by integrating in $t$.

\section{APPENDIX C. - EXCLUSION OF GRADIENT BLOW-UP: PROOF OF PROPOSITION 2.1}

We need two preparatory lemmas. All the constants $C_{i}$ in the statements of Lemmas $\mathrm{C} 1$ and $\mathrm{C} 2$ remain bounded when their arguments remain in a bounded set. The first lemma shows that blow-up in any $L^{r}$ norm (for large $r \leq \infty)$ implies blow-up in all of these norms. Throughout this section, $u(t)$ is the solution of (A2) with initial value $\phi \in W_{0}^{1, s}(\Omega)$, as in Appendix A. Here, we need to set $J_{1}(u)=-\mu|\nabla u|^{q}, \mu>0$, and $J_{2}(u)=|u|^{p-1} u$.

LEMMA C1. - Let $\phi \in W_{0}^{1, s}(\Omega), \phi \geq 0, s \leq r<\infty$ and $0<T<$ $T^{*} \leq \infty$. Then

$$
\sup _{0 \leq t \leq T}\|u(t)\|_{r} \leq C_{1}\left(T,\|\phi\|_{r}, \sup _{0 \leq t \leq T}\|u(t)\|_{\infty}\right)
$$

If, in addition, $r>\max (p, N p / 2)$, we have

$$
\sup _{0 \leq t \leq T}\|u(t)\|_{\infty} \leq C_{2}\left(T,\|\phi\|_{\infty}, \sup _{0 \leq t \leq T}\|u(t)\|_{r}\right)
$$

Proof of Lemma C1. - Let us first assume that $\phi \in C_{c}^{3}(\Omega)$, so that (2.1) holds. For any finite $r \geq s$, multiplying (1.1) by $u^{r-1}$, integrating on $\Omega$ 
and using Green's formula yields

$$
\begin{aligned}
\frac{d}{d t} \int \frac{u^{r}}{r} & =\int u^{r-1} \Delta u+\int u^{p+r-1}-\mu \int u^{r-1}|\nabla u|^{q} \\
& =-(r-1) \int u^{r-2}|\nabla u|^{2}+\int u^{p+r-1}-\mu \int u^{r-1}|\nabla u|^{q} \\
& \leq \sup _{0 \leq t \leq T}\|u(t)\|_{\infty}^{p-1} \int u^{r},
\end{aligned}
$$

hence,

$$
\|u(t)\|_{r} \leq\|u(0)\|_{r} \exp \left[r T \sup _{0 \leq t \leq T}\|u(t)\|_{\infty}^{p-1}\right] .
$$

By density and continuous dependence, using the embedding $W^{1, s} \subset L^{\infty}$, this inequality holds in fact for any $\phi \in W_{0}^{1, s}(\Omega)$ and (C1) follows.

Next, it follows from the integral equation

$$
u(t)=c^{t \Delta} u(0)+\int_{0}^{t} e^{(t-\tau) \Delta}\left(u^{p}(\tau)-\mu|\nabla u(\tau)|^{q}\right) d \tau,
$$

the nonnegativeness of $u$, property (SG4), and $r>\max (p, N p / 2)$, that for all $t \in[0, T]$,:

$$
\begin{aligned}
\|u(t)\|_{\infty} & \leq\|u(0)\|_{\infty}+C \sup _{0 \leq t \leq T}\|u(s)\|_{r}^{p} \int_{0}^{t}(t-s)^{-N p / 2 r} d s \\
& \leq\|u(0)\|_{\infty}+C(1+T)^{1-N p / 2 r} \sup _{0 \leq t \leq T}\|u(s)\|_{r}^{p},
\end{aligned}
$$

and the result follows.

Remark $\mathrm{C} 1$. - By an iteration procedure, one can see that the second part of Lemma $\mathrm{Cl}$ (and hence Proposition 2.1) remains valid in fact for all $r \geq s$, with $r \geq p$ and $r>N(p-1) / 2$.

The next lemma, which is modeled after [Q2, Theorem 5.1 (i)], shows that the absence of blow-up of $u$ in $L^{\infty}$ norm prevents blow-up of the gradient of $u$ in $L^{\infty}$ norm.

Lemma C2. - Let $\phi \in W_{0}^{1, s}(\Omega), \phi \geq 0$. There exists some $t_{0}=$ $t_{0}\left(\|\phi\|_{W^{1, s}}\right)>0$ such that $T^{*}>t_{0}$, and such that for all $T, t_{0}<T<T^{*}$,

$$
\sup _{t_{0} \leq t \leq T}\|\nabla u(t)\|_{\infty} \leq C_{3}\left(T,\|\phi\|_{W^{1, s}}, \sup _{t_{0} \leq t \leq T}\|u(t)\|_{\infty}\right) .
$$

Proof of Lemma C2. - We first assume that $\phi \in C_{c}^{3}(\Omega)$ and define the function $w(t, x)=\frac{1}{2}|\nabla u(t, x)|^{2}$. By Propositions $\mathrm{A} 3$ and $\mathrm{A} 4$, it follows that

$$
w \in C^{1}\left(\left[0, T^{*}\right), L^{s}(\Omega)\right) \text { and } w \in C\left(\left[0, T^{*}\right), W^{2, s}(\Omega)\right) .
$$

Vol. $16, \mathrm{n}^{\circ} 3-1999$ 
Moreover, $w$ satisfies the equation

$$
w_{t}=\Delta w-\sum_{i, j}\left(\partial_{x_{i, x_{j}}} w\right)^{2}+2 p u^{p-1} w-\mu q|\nabla u|^{q-2} \nabla u . \nabla w .
$$

where each term is in $C\left(\left[0, T^{*}\right), L^{s}(\Omega)\right)$. By Proposition A2 (ii), there exists $t_{0}=t_{0}\left(\|\phi\|_{W^{1, s}}\right)>0$ such that $T^{*}>t_{0}$, and such that

$$
\left\|\nabla u\left(t_{0}\right)\right\|_{\infty} \leq C_{0}\left(\|\phi\|_{W^{1, s}}\right)
$$

Fix $T \in\left(t_{0}, T^{*}\right)$, and set

$$
A=2 p \sup _{t_{0} \leq t \leq T}\|u(t)\|_{\infty}^{p-1}<\infty \text { and } B=\mu q \sup _{t_{0} \leq t \leq T}\|\nabla u(t)\|_{\infty}^{q-1}<\infty .
$$

Then the function $z(t, x)=w(t, x) \exp \left(A\left(t_{0}-t\right)\right)$ satisfies the parabolic inequality

$$
z+\leq z+B|\nabla z|
$$

where each term is in $C\left(\left[t_{0}, T\right], L^{s}(\Omega)\right)$. Let $Q=\left(t_{0}, T\right) \times \Omega$. Since $z \in$ $C\left(\left[t_{0}, T\right], W^{1, s}(\Omega)\right)$, it follows from (A6) and a compactness argument that the supremum of $z$ in $\bar{Q}$ is attained at some point of $\bar{Q}$. Applying the weak comparison principle (see Proposition B2), we deduce that the maximum $Z$ of $z$ is attained on the parabolic boundary $\left(\left\{t_{0}\right\} \times \Omega\right) \cup\left(\left(t_{0}, T\right) \times \partial \Omega\right)$. The end of the argument now proceeds exactly as in [Q2, Theorem 5.1, p. 118]. We give the details for the convenience of the reader.

If the maximum $Z$ is in fact attained on $\left\{t_{0}\right\} \times \Omega$, then (C4) immediately gives

$$
\sup _{t_{0} \leq t \leq T}\|\nabla u(t)\|_{\infty} \leq C_{0}\left(\|\phi\|_{H^{-\ldots .}}\right) \exp \left(A\left(T-t_{0}\right)\right) .
$$

If $Z=z\left(t, x_{0}\right)$ for some point $\left(t, x_{0}\right) \in\left(t_{0}, T\right) \times \partial \Omega$, then, since $u$ is a classical solution up to the boundary (Corollary A5), it follows that

$$
0=u_{t}\left(t, x_{0}\right)=\Delta u\left(t, x_{0}\right)-\mu\left|\nabla u\left(t, x_{0}\right)\right|^{q} .
$$

By Lemma C3 (see after the end of this proof), we have

$$
\left|\Delta u-\frac{\partial^{2} u}{\partial n^{2}}\left(t, x_{0}\right)\right| \leq N C(\Omega)\left|\frac{\partial u}{\partial n}\left(t, x_{0}\right)\right|=N C(\Omega)\left|\nabla u\left(t, x_{0}\right)\right|
$$

Since $|\nabla u(t,)$.$| attains its maximum at \left(t, x_{0}\right)$, we have $\frac{\partial^{2} u}{\partial u^{2}}\left(t, x_{0}\right) \leq 0$. Therefore, by (C6) and (C7), we obtain $\left|\nabla u\left(t, x_{0}\right)\right|^{q-1} \leq N C(\Omega) / \mu$. Both this inequality and (C5) give the desired estimate (C3). Proposition A2 and 
the embedding $W_{0}^{1, s}(\Omega) \subset L^{\infty}(\Omega)$ allow one to extend this by density to all $\phi \in W_{0}^{1, s}(\Omega)$.

The previous proof made use of the following geometric lemma. This property is stated without proof in the work of Quittner [Q2]. Though it is of an elementary nature, we prefer to provide a proof here, both for sake of completeness, and because the required assumptions on $\Omega$ have to be made precise when dealing with an unbounded domain.

Lemma C3. - Assume that $\Omega$ is uniformy regular of class $C^{2}$ (see Appendix A). Then for any function $v \in C^{2}(\bar{\Omega})$, vanishing on $\partial \Omega$, it follows that

$$
\left|\frac{\partial^{2} v}{\partial \tau^{2}}\left(x^{0}\right)\right| \leq C(\Omega)\left|\frac{\partial v}{\partial n}\left(x^{0}\right)\right|
$$

for all $x^{0} \in \partial \Omega$, where $n$ is the normal direction and $\tau$ any tangential direction to $\partial \Omega$ at $x^{0}$.

Proof of Lemma C3. - Let $x^{0}$ be a point of $\partial \Omega$. By the uniform regularity assumption, there is a parametrization of $\partial \Omega$ in a neighborhood $U$ of $x^{0}$, of the form $x_{N}=\theta\left(x^{\prime}\right)=\theta\left(x_{1}, \cdots, x_{N-1}\right)$, satisfying $\left\|D^{2} \theta\right\|_{\infty} \leq C(\Omega)$. Let $\left(e_{i}\right)_{1 \leq i \leq N}$ be the corresponding orthonormal basis of $\mathbb{R}^{N}$.

Without loss of generality (by an orthogonal change of coordinates), one can assume that the tangent hyperplane to $\partial \Omega$ at $x^{0}$ is parallel to $e_{1}, \cdots, e_{N-1}$, and that the exterior normal is parallel to $e_{N}$. Let $1 \leq i \leq N-1$. The $i^{\text {th }}$ tangent vector to $\partial \Omega$ at a point $x \in \partial \Omega \cap U$ is given by

$$
\tau_{i}(x)=\left[1+\left(\frac{\partial \theta}{\partial x_{i}}\right)^{2}\left(x^{\prime}\right)\right]^{-1 / 2}\left(e_{i}+\frac{\partial \theta}{\partial x_{i}}\left(x^{\prime}\right) e_{N}\right) .
$$

Since $v(x)=0$ on $\partial \Omega$, it follows that

$$
\nabla v(x) \cdot \tau_{i}(x)=0
$$

for all $x \in \partial \Omega \cap U$. In other words,

$$
\nabla v(x) \cdot e_{i}=-\frac{\partial \theta}{\partial x_{i}}\left(x^{\prime}\right) \nabla v(x) \cdot e_{N} .
$$

On the other hand,

$$
\begin{gathered}
\frac{\partial^{2} v}{\partial x_{i}^{2}}\left(x^{0}\right)=\lim \frac{\left(\nabla v(x)-\nabla v\left(x^{0}\right)\right) \cdot e_{i}}{\left|x-x^{0}\right|} \text { as } x \rightarrow x^{0}, \\
\text { with } x \in \partial \Omega, \text { and } \frac{x-x^{0}}{\left|x-x^{0}\right|} \cdot e_{i} \rightarrow 1 .
\end{gathered}
$$

Vol. 16, n 3-1999. 
Since $\frac{\partial \theta}{\partial x_{i}}\left(x^{0^{\prime}}\right)=0$ and $\nabla v\left(x^{0}\right) \cdot e_{i}=0$, we infer from $(\mathrm{C} 8)$ and the mean-value theorem that

$$
\left|\frac{\partial^{2} v}{\partial x_{i}^{2}}\left(x^{0}\right)\right| \leq\left\|D^{2} \theta\right\|_{\infty}\left|\frac{\partial v}{\partial x_{N}}\left(x^{0}\right)\right|,
$$

and the result follows.

Completion of proof of Proposition 2.1. - Assume that $T^{*}<\infty$ and

$$
\limsup _{t \rightarrow T^{*}}\|u(t)\|_{r}<\infty
$$

for some $r$ such that $s \leq r \leq \infty$ and $r>\max (p, N p / 2)$. Then, by Lemma $\mathrm{C} 1$, (C9) actually holds for any $r, s \leq r \leq \infty$. By Lemma $\mathrm{C} 2$, since $C_{3}$ is bounded function of its arguments, we then obtain

$$
\limsup _{t \rightarrow T^{*}}\|u(t)\|_{W^{1, \infty}}<\infty .
$$

By Proposition A2, this implies that $T^{*}=\infty$, a contradiction.

\section{ACKNOWLEDGEMENTS}

The authors wish to thank H. Amann, M. Balabane, M. Escobedo, and E. Lieb for their interest in this work and helpful suggestions.

\section{REFERENCES}

[Ad] R. A. Adams, Sobolev Spaces, Academic Press, New York, 1975.

[AE] J. AguirRe and M. EscoBedo, On the blow up of solutions for a convective reaction diffusion equation, Proc. Roy. Soc. Edinburgh., Vol. 123, 1993, pp. 433-460.

[Am] H. Amann, Existence and regularity for semilinear parabolic evolution equations, Ann. Scuola Norm. Sup. Pisa, Vol. 11, 4, 1984, pp. 593-676.

[AW] L. ALFONSI and F. B. WEISSLER, blow-up in $\mathbb{R}^{N}$ for a parabolic equation with a damping nonlinear gradient term, Progress in nonlinear differential equations 1992, N. G. Lloyd et al. Eds, Birkhäuser.

[BC] H. BREZIS and T. CAZENAVE, to appear.

[B] F. E. BRowder, On the spectral theory of elliptic operators, I, Math. Ann., Vol. 142, 1961, pp. 22-30.

[CW] M. CHIPOT and F. B. WEISSLER, Some blow up results for a nonlinear parabolic problem with a gradient term, SIAM J. Math. Anal., Vol. 20, 4, 1989, pp. 886-907.

[E] M. Escobedo, Personal communication.

[F] M. FILA, Remarks on blow up for a nonlinear parabolic equation with a gradient term, Proc. Amer. Math. Soc., Vol. 111, 2, 1991, pp. 795-801. 
[Fr1] A. Friedman, Partial Differential Equations, 1969, Holt, Rinehart and Winston, Inc., New York.

[Fr2] A. Friedman, Blow up of solutions of parabolic equations, Nonlinear diffusion equations and their equilibrium states, I 1988, W. M. Ni et al. Eds, Springer.

[H] W. HaYman, Some bounds for principal frequency, Applicable Analysis, Vol. 7, 1978. pp. 247-254.

[KP] B. Kawohl and L. A. Peletier, Observations on blow up and dead cores for nonlinear parabolic equations, Math. Z., Vol. 202, 1989, pp. 207-217.

[LSU] O. A. Ladyzenskaja, V. A. Solonnikov and N. N. Uralceva, Linear and Quasilinear Equations of Parabolic Type, 1968, Translations of Mathematical Monographs, Amer. Math. Soc., Providence, RI.

[LN] T. LEE and W. NI, Global existence, large time behaviour and life span of solutions of a semilinear parabolic Cauchy problem, Trans. Amer. Math. Soc., Vol. 333, 1, 1992. pp. 365-378.

[LPSS] H. A. Levine, L. N. Payne, P. E. Sacks and B. Straughan, Analysis of convective reaction-diffusion equation (II), SIAM J. Math. Anal, Vol. 20, 1, 1989, pp. 133-147.

[L] E. A. LIEB, On the lowest eigenvalue of the Laplacian for the intersection of two domains, Invent. Math., Vol. 74, 1983, pp. 441-448.

[Q1] P. QuITTNER, Blow-up for semilinear parabolic equations with a gradient term, Math. Meth. Appl. Sc., Vol. 14, 1991, pp. 413-417.

[Q2] P. QUITTNER, On global existence and stationary solutions for two classes of semilinear parabolic equations, Comment. Math. Univ. Carolinae, Vol. 34, 1, 1993, pp. 105-124.

[O] R. Osserman, A note on Hayman's theorem on the bass note of a drum. Comment. Math. Helvetici, Vol. 52, 1 1977, pp. 545-555.

[S1] P. Souplet, Résultats d'explosion en temps fini pour une équation de la chaleur non linéaire, C. R. Acad. Sc. Paris, Vol. 321, Série I, 1995, pp. 721-726.

[S2] P. Souplet, Finite time blow up for a nonlinear parabolic equation with a gradient term and applications, Math. Meth. Appl. Sc., Vol. 19, 1996, pp. 1317-1333.

[S3] P. Souplet, Geometry of unbounded domains, Poincaré inequalities and stability in semilinear parabolic equations, Communications in Partial Differential Equations, to appear.

[STW] P. SOUPlet, S. TAYACHI and F. B. Weissler, Exact self-similar blow-up of solutions of a semilinear parabolic equation with a nonlinear gradient term, Indiana Univ. Math. J., Vol. 48, 3, 1996, pp. 655-682.

[SW] P. SOUPLET and F. B. WeisSLER, Self-similar sub-solutions and blow-up for nonlinear parabolic equations, J. Math. Anal. Appl., Vol. 212, 1997, pp. 60-74.

[W] F. B. WeISSLER, Semilinear evolution equations in Banach spaces, J. Functional Analysis, Vol. 32, 4, 1979, pp. 277-296. 